\title{
TORSION IN STRAIN-GRADIENT PLASTICITY: ENERGETIC SCALE EFFECTS*
}

\author{
MARIA CHIRICOTTO ${ }^{\dagger}$, LORENZO GIACOMELLI $^{\dagger}$, AND GIUSEPPE TOMASSETTI ${ }^{\ddagger}$
}

\begin{abstract}
We study elasto-plastic torsion in a thin wire within the framework of the straingradient plasticity theory elaborated by Gurtin and Anand in 2005. The theory in question envisages two material scales: an energetic length-scale, which takes into account the so-called "geometricallynecessary dislocations" through a dependence of the free energy on the Burgers tensor, and a dissipative length-scale. For the rate-independent case with null dissipative length-scale, we construct and characterize a special class of solutions to the evolution problem. With the aid of such characterization, we estimate the dependence on the energetic scale of the ratio between the torque and the twist. Our analysis confirms that the energetic scale is responsible for size-dependent strain-hardening, with thinner wires being stronger. We also detect, and quantify in terms of the energetic length-scale, both a critical twist, after which the wire becomes fully plastified, and two boundary layers near the external boundary of the wire and near the boundary of the plastified region, respectively.
\end{abstract}

Key words. size effects, Burgers vector, rate-independent evolution, elasto-plastic boundary

AMS subject classifications. $74 \mathrm{C} 05,35 \mathrm{~K} 85,34 \mathrm{E} 10$

DOI. $10.1137 / 120863034$

\section{Introduction and results.}

1.1. The torsion experiment. At the micron scale, metallic components undergoing nonuniform plastic flow are known to display size-dependent behavior: generally speaking, "smaller" specimens appear to be "stronger." Among the many examples, of particular interest to us is a series of torsion experiments, reported in [13], on copper wires of equal length and diameter ranging from 170 down to $12 \mu \mathrm{m}$. In these experiments, the wires were twisted (monotonically, and with the same rate) well into the plastic range, and the relationship between torque $Q$ and twist $\Theta$ (the angle of rotation per unit length) was recorded. Had the wires been governed by a continuum theory with no constitutive length parameter, such as conventional plasticity, the torque $Q$ needed to impart a twist $\Theta$ to a wire of radius $R$ should obey

$$
\frac{Q}{R^{3}}=f(R \Theta)
$$

where the function $f(\cdot)$ depends only on the material constituting the wire. Instead, the experimental curves reported in [13] violate (1.1), and show that thinner wires have higher relative strength, in the sense that a higher specific work input, $Q / R^{3}$, is needed to induce the same strain, $R \Theta$, in a thinner wire.

The inability of conventional plasticity to capture size effects is alleviated by strain-gradient plasticity theories through an explicit appearance of the plastic-strain gradient in the field equations $[2,3,7,8,11,13,17,20,21]$. Here we are concerned with small-strain theories, and we focus on a theory for isotropic materials developed

\footnotetext{
* Received by the editors January 23, 2012; accepted for publication (in revised form) May 8, 2012; published electronically August 21, 2012.

http://www.siam.org/journals/siap/72-4/86303.html

†Dipartimento SBAI, Università di Roma "La Sapienza," 00161 Rome, Italy (maria.chiricotto@ sbai.uniroma1.it, lorenzo.giacomelli@sbai.uniroma1.it).

¥Dipartimento di Ingegneria Civile, Università di Roma "Tor Vergata," 00133 Rome, Italy (tomassetti@ing.uniroma2.it). This author thanks the Italian INdAM-GNFM for financial support.
} 
by Gurtin and Anand in [21]. For additional details, we refer to the recent monograph [22], where the theory is expounded.

1.2. Conventional plasticity. For the reader's convenience, we begin by recalling the field equations from standard small-strain plasticity theory for isotropic materials, with specific reference to flow theories commonly used for metals. In smallstrain plasticity, the unknowns are the displacement $\mathbf{u}(x, t) \in \mathbb{R}^{3}$ and the plastic strain $\mathbf{E}^{\mathrm{P}}(x, t) \in \mathbb{R}_{0, \text { sym }}^{3 \times 3}$. The elastic strain $\mathbf{E}^{\mathrm{e}}(x, t) \in \mathbb{R}_{\text {sym }}^{3 \times 3}$, defined by ${ }^{1}$

$$
\mathbf{E}^{\mathrm{e}}:=\operatorname{sym} \nabla \mathbf{u}-\mathbf{E}^{\mathrm{p}},
$$

determines the stress $\mathbf{T}(x, t) \in \mathbb{R}_{\text {sym }}^{3 \times 3}$ through the constitutive equation

$$
\mathbf{T}=\frac{\partial \psi_{\mathrm{e}}}{\partial \mathbf{E}^{\mathrm{e}}}, \quad \psi_{\mathrm{e}}\left(\mathbf{E}^{\mathrm{e}}\right):=\mu\left|\mathbf{E}_{0}^{\mathrm{e}}\right|^{2}+\frac{1}{2} \kappa\left|\operatorname{tr} \mathbf{E}^{\mathrm{e}}\right|^{2},
$$

where $\psi_{\mathrm{e}}\left(\mathbf{E}^{\mathrm{e}}\right)$ is the elastic energy density and $\mu, \kappa>0$. When body forces are null, the stress obeys the force balance:

$$
\operatorname{div} \mathbf{T}=0
$$

Flow rules adopted in small-strain metal plasticity typically have the form

$$
\mathbf{T}_{0}=Y\left(e^{\mathrm{p}}\right) g\left(\left|\dot{\mathbf{E}}^{\mathrm{p}}\right|\right) \frac{\dot{\mathbf{E}}^{\mathrm{p}}}{\left|\dot{\mathbf{E}}^{\mathrm{p}}\right|}
$$

where $Y(\cdot)>0$ is the flow resistance, $e^{\mathrm{p}}(x, t)=\int_{0}^{t}\left|\dot{\mathbf{E}}^{\mathrm{p}}(x, \mathfrak{t})\right| \mathrm{d} \mathbf{t}$ is the accumulated plastic strain, and $g(\cdot)$ is a (dimensionless) rate-sensitivity function. The simplest choice for the rate-sensitivity function is the power law $g\left(\left|\dot{\mathbf{E}}^{\mathrm{p}}\right|\right)=\left(\frac{\left|\dot{\mathbf{E}}^{\mathrm{p}}\right|}{d_{0}}\right)^{m}$, where $d_{0}>0$ is a reference rate and the parameter $m \geq 0$ is a measure of rate dependency: for $m=0$, we have $g(\cdot)=1$, and the flow rule (1.3) is not affected by a monotone time reparametrization.

1.3. The Gurtin-Anand model. The inability of (1.2)-(1.3) to capture size effects is due to its invariance under the scaling $x \mapsto \alpha x, \mathbf{u} \mapsto \alpha \mathbf{u}(\alpha>0)$. In [21, 22], size dependence is achieved by replacing (1.3) with a flow rule that accounts for the plastic-strain gradient in two ways:

\footnotetext{
${ }^{1}$ Boldface lower case $\left(\mathbf{a}, \mathbf{b}\right.$, etc.) denotes elements of $\mathbb{R}^{3}$ (vectors), boldface upper case $(\mathbf{A}, \mathbf{B}$, etc.) denotes elements of $\mathbb{R}^{3 \times 3}$ (tensors), and blackboard bold upper case $(\mathbb{A}, \mathbb{B}$, etc.) denotes elements of $\mathbb{R}^{3 \times 3 \times 3}$ (second-order tensors). We denote the components of $\mathbf{a}, \mathbf{A}$, and $\mathbb{A}$ in the corresponding standard basis by $(\mathbf{a})_{i},(\mathbf{A})_{i j}$, and $(\mathbb{A})_{i j k}$, respectively. We use the summation convention. We let $\mathbf{a} \cdot \mathbf{b}=(\mathbf{a})_{i}(\mathbf{b})_{i}, \mathbf{A}: \mathbf{B}=(\mathbf{A})_{i j}(\mathbf{B})_{i j}, \mathbb{A}: \mathbb{B}=(\mathbb{A})_{i j k}(\mathbb{B})_{i j k},(\mathbf{A a})_{j}=(\mathbf{A})_{i j}(\mathbf{a})_{j},(\mathbb{A} \mathbf{a})_{i j}=(\mathbb{A})_{i j k}(\mathbf{a})_{k}$. We denote by $\mathbb{R}_{s y m}^{3 \times 3}$ and $\mathbb{R}_{0}^{3 \times 3}$ the sets of symmetric, respectively, traceless, second-order tensors, and we let $\mathbb{R}_{0, \text { sym }}^{3 \times 3}=\mathbb{R}_{0}^{3 \times 3} \cap \mathbb{R}_{s y m}^{3 \times 3}$. Likewise we denote by $\mathbb{R}_{s y m}^{3 \times 3 \times 3}$ and $\mathbb{R}_{0}^{3 \times 3 \times 3}$ the set of thirdorder tensors that are symmetric, respectively, deviatoric, with respect to the first two indices, and we let $\mathbb{R}_{0, s y m}^{3 \times 3 \times 3}=\mathbb{R}_{0}^{3 \times 3 \times 3} \cap \mathbb{R}_{s y m}^{3 \times 3 \times 3}$. We denote by $\mathbf{a} \otimes \mathbf{b}$, respectively, $\mathbf{a} \otimes \mathbf{b} \otimes \mathbf{c}$, the secondorder, respectively, third-order, tensor defined componentwise by $(\mathbf{a} \otimes \mathbf{b})_{i j}=(\mathbf{a})_{i}(\mathbf{b})_{j}$, respectively, $(\mathbf{a} \otimes \mathbf{b} \otimes \mathbf{c})_{i j k}=(\mathbf{a})_{i}(\mathbf{b})_{j}(\mathbf{c})_{k}$. We denote by symA and $\mathbf{A}_{0}$, respectively, the symmetric part and deviatoric part of any tensor $\mathbf{A}$, namely, $\operatorname{sym} \mathbf{A}=\frac{1}{2}\left(\mathbf{A}^{T}+\mathbf{A}\right)$ and $\mathbf{A}_{0}=\mathbf{A}-\frac{1}{3} \operatorname{tr}(\mathbf{A}) \mathbf{I}$, where $\mathbf{I}$ is the identity matrix. Given scalar functions $f$ and $g$ depending on $\mathbf{A}$, respectively, $\mathbb{A}$, we use the notation $\frac{\partial f}{\partial \mathbf{A}}$ and $\frac{\partial g}{\partial \mathbb{A}}$ to denote the second-order, respectively, third-order, tensors defined by $\left(\frac{\partial f}{\partial \mathbf{A}}\right)_{\langle i j\rangle}=\frac{\partial f(\mathbf{A})}{\partial \mathbf{A}_{\langle i j\rangle}},\left(\frac{\partial g}{\partial \mathbb{A}}\right)_{\langle i j k\rangle}=\frac{\partial g(\mathbb{A})}{\partial \mathbb{A}_{\langle i j k\rangle}}$.
} 
(1) an energetic scale dependence, and a corresponding energetic length-scale $L$, are introduced by adding to the elastic energy density a defect energy density

$$
\psi_{\mathrm{d}}\left(\nabla \mathbf{E}^{\mathrm{p}}\right)=\frac{1}{2} \mu L^{2}\left((1-\eta)\left|\operatorname{curl} \mathbf{E}^{\mathrm{p}}\right|^{2}+\eta\left|\operatorname{curl} \mathbf{E}^{\mathrm{p}}-\left(\operatorname{curl} \mathbf{E}^{\mathrm{p}}\right)^{T}\right|^{2}\right)
$$

(cf. [22, eqs. (90.41)-(90.42)] with $\lambda_{2}=\eta$ ), where $-1<\eta<1$ is a dimensionless parameter. In the framework of the Gurtin-Anand theory, curlE $\mathbf{E}^{\mathrm{p}}$ coincides with the Burgers tensor [22, section 88.1-2], which provides a macroscopic description of geometrically necessary dislocations.

(2) a dissipative scale dependence, and a corresponding dissipative length-scale $\ell$, are introduced by a dependence of the dissipation-rate density on spatial derivatives of the plastic strain-rate, $\dot{\mathbf{E}}^{\mathrm{p}}$. The dissipation-rate density is given by

$$
\begin{array}{r}
\delta=Y\left(E^{\mathrm{p}}\right) g\left(d^{\mathrm{p}}\right) d^{\mathrm{p}}, \quad \text { where } \quad d^{\mathrm{p}}:=\sqrt{\left|\dot{\mathbf{E}}^{\mathrm{p}}\right|^{2}+\ell^{2}|\nabla \dot{\mathbf{E}}|^{2}} \\
\text { and } E^{\mathrm{p}}(x, t):=\int_{0}^{t} d^{\mathrm{p}}(x, s) \mathrm{d} s .
\end{array}
$$

More specifically, Gurtin and Anand replace (1.3) with the microforce balance

$$
\mathbf{T}_{0}=\mathbf{T}^{\mathrm{p}}-\operatorname{div} \mathbb{K}^{\mathrm{p}}
$$

and with the following constitutive equations for the plastic stress $\mathbf{T}^{\mathrm{p}}(x, t) \in \mathbb{R}_{0, \text { sym }}^{3 \times 3}$ and the plastic microstress $\mathbb{K}^{\mathrm{p}}(x, t) \in \mathbb{R}_{0, \text { sym }}^{3 \times 3 \times 3}$ :

$$
\mathbf{T}^{\mathrm{p}}=Y\left(E^{\mathrm{p}}\right) g\left(d^{\mathrm{p}}\right) \frac{\dot{\mathbf{E}^{\mathrm{p}}}}{d^{\mathrm{p}}}, \quad \mathbb{K}^{\mathrm{p}}=\mathbb{K}_{\mathrm{en}}^{\mathrm{p}}+\mathbb{K}_{\mathrm{diss}}^{\mathrm{p}}, \quad\left\{\begin{array}{l}
\mathbb{K}_{\mathrm{en}}^{\mathrm{p}}=\frac{\partial \psi_{\mathrm{d}}}{\partial \nabla \mathbf{E}^{\mathrm{p}}} \\
\mathbb{K}_{\mathrm{diss}}^{\mathrm{p}}=\ell^{2} Y\left(E^{\mathrm{p}}\right) g\left(d^{\mathrm{p}}\right) \frac{\nabla \dot{\mathbf{E}}^{\mathrm{p}}}{d^{\mathrm{p}}}
\end{array}\right.
$$

1.4. The goals. Quite a lot of effort has been put into the mathematical analysis of this theory: besides [30], which deals with the (much more tractable) case in which hardening is present, in [16] the concept of "energetic solution" (see [25]) is implemented for this model in the rate-independent case (which follows by formally substituting $g(\cdot)=1$ in $(1.5 \mathrm{~b})$ ). However, we are not aware of analytical studies aiming to qualify and quantify the scale effects induced by $\ell$ and $L$. To the best of our knowledge, only dimensional and numerical observations are available so far $[20,4]$, suggesting the following:

(a) the development of boundary layers near $\partial \Omega$, at least in the case of no flux of the Burgers vector through $\partial \Omega$ [20, section 10.2],

(b) an increase of the strain-hardening rate with $L[4$, section 12],

(c) an increase of the strengthening with $\ell[4$, section 12].

The goal of the present paper is to obtain a more robust validation of the role of the energetic length-scale $L$ with respect to the observations in (a) and (b). To this end: (1) we assume constant flow resistance and we rule out dissipative size effects by setting

$$
\begin{aligned}
& Y(\cdot)=\sqrt{2} k, \\
& \ell=0, \quad \text { i.e., } \quad d^{\mathrm{p}}=\left|\dot{\mathbf{E}}^{\mathrm{p}}\right|,
\end{aligned}
$$

where $k>0$ is the yield strength under pure shear; $(2)$ we take the rate-independent limit $g(s)=1$, so that $\delta\left(\dot{\mathbf{E}}^{\mathrm{p}}\right)=\sqrt{2} k\left|\dot{\mathbf{E}}^{\mathrm{p}}\right|$, and we replace the first equation of $(1.5 \mathrm{~b})$ 
with

$$
\begin{cases}\mathbf{T}^{\mathrm{p}} \in \mathbb{R}_{0, \text { sym }}^{3 \times 3} \text { and }\left|\mathbf{T}^{\mathrm{p}}\right| \leq \sqrt{2} k & \text { if } \dot{\mathbf{E}}^{\mathrm{p}}=0, \\ \mathbf{T}^{\mathrm{p}}=\sqrt{2} k \frac{\dot{\mathbf{E}}^{\mathrm{p}}}{\left|\dot{\mathbf{E}}^{\mathrm{p}}\right|} & \text { if } \quad \dot{\mathbf{E}}^{\mathrm{p}} \neq 0 .\end{cases}
$$

It is not hard to check that (1.6c) is equivalent to the following differential inclusion [23]:

$(1.7)$

$\mathbf{T}^{\mathrm{p}} \in \partial \delta\left(\dot{\mathbf{E}}^{\mathrm{p}}\right)=\left\{\mathbf{A} \in \mathbb{R}_{0, s y m}^{3 \times 3}: \delta\left(\widetilde{\mathbf{E}}^{\mathrm{p}}\right)-\delta\left(\dot{\mathbf{E}}^{\mathrm{p}}\right) \geq \mathbf{A}:\left(\widetilde{\mathbf{E}}^{\mathrm{p}}-\dot{\mathbf{E}}^{\mathrm{p}}\right)\right.$ for all $\left.\widetilde{\mathbf{E}}^{\mathrm{p}} \in \mathbb{R}_{0, s y m}^{3 \times 3}\right\}$.

Using (1.6) and (1.4), after an explicit computation of $\mathbb{K}^{\mathrm{p}}$ (see section 2) the flow rule (1.5) becomes

$$
\mathbf{T}_{0}+\mu L^{2}\left(\Delta \mathbf{E}^{\mathrm{p}}-\operatorname{sym}\left(\nabla \operatorname{div} \mathbf{E}^{\mathrm{p}}\right)+\frac{1}{3}(1+\eta)\left(\operatorname{div} \operatorname{div} \mathbf{E}^{\mathrm{p}}\right) \mathbf{I}+\eta \operatorname{curlcurl} \mathbf{E}^{\mathrm{p}}\right) \in \partial \delta\left(\dot{\mathbf{E}}^{\mathrm{p}}\right) .
$$

Note that when $L=0$, the flow rule (1.8) reduces to (1.7) with $\mathbf{T}^{\mathrm{p}}$ replaced by $\mathbf{T}_{0}$; the resulting law characterizes, according to the terminology of [22], the Levy-Mises plastic response.

Though the effects in (a) and (b) already seem to be observable, at least qualitatively, at the level of the one-dimensional theory proposed in [4], we wish to explore them in a multidimensional setting where the role of the Burgers tensor (as the curl of a tensor field) should become more transparent on the one hand and experimental results are available on the other hand. One such setting is, of course, that of the torsion problem, which has already been studied in the context of other strain-gradient plasticity theories $[13,17,7]$, and which we introduce now.

1.5. The torsion problem. We model a thin metallic wire as an infinite rightcylinder

$$
\Omega_{R}=\left\{x=(\varrho \cos \phi, \varrho \sin \phi, z) \in \mathbb{R}^{3}: \varrho \in[0, R), \phi \in[0,2 \pi)\right\}
$$

subject to null tractions at the boundary $\partial \Omega_{R}\left(\right.$ see $\left.(2.13)_{2}\right)$. Denoting by $\left(\mathbf{e}_{\langle 1\rangle}, \mathbf{e}_{\langle 2\rangle}, \mathbf{e}_{\langle 3\rangle}\right)$ the local orthonormal frame (see (2.1)) associated with the cylindrical coordinates $(\varrho, \phi, z)$, we write the following Ansatz:

$$
\begin{aligned}
& \mathbf{u}(\varrho, \phi, z, t)=z \Theta(t) \varrho \mathbf{e}_{\langle 2\rangle}(\phi), \\
& \mathbf{E}^{\mathrm{p}}(\varrho, \phi, t)=\gamma^{\mathrm{P}}(\varrho, t) \operatorname{sym}\left(\mathbf{e}_{\langle 2\rangle}(\phi) \otimes \mathbf{e}_{\langle 3\rangle}\right),
\end{aligned}
$$

where both the twist $\Theta$ and the plastic-shear profile $\gamma^{\mathrm{p}}$ satisfy the null initial conditions:

$$
\Theta(0)=0, \quad \gamma^{\mathrm{p}}(\cdot, 0)=0 .
$$

The stress field that results from (1.10) and (1.2a-1.2b) satisfies the balance equation (1.2c) and the null-traction condition for the standard forces. Moreover, as we shall see in section 2.2, the torque that must be applied to induce a twist $\Theta$ with plastic-shear profile $\gamma^{\mathrm{p}}$ is given by the following expression:

$$
Q=2 \pi \mu \int_{0}^{R}\left(\Theta \varrho-\gamma^{\mathrm{p}}\right) \varrho^{2} \mathrm{~d} \varrho .
$$

An important point to be made at first is that the system (1.8) is unaffected by a monotone time reparametrization. This property is best exploited when the twist 
is monotone: $\dot{\Theta}>0$, an additional working assumption that we make in this paper. Indeed, this assumption enables us to replace the dependence of $\gamma^{\mathrm{p}}$ on time with a dependence on the twist by performing the substitution: $\gamma^{\mathrm{P}}(\varrho, t) \mapsto \gamma^{\mathrm{P}}(\varrho, \Theta)$. A second point is that, since the system has no intrinsic timescale, the only parameter that matters is the ratio between the energetic length-scale $L$ and the diameter $2 R$. To highlight the role of this parameter, we introduce a normalized energetic lengthscale $\lambda$, proportional to $L / R$ (see (4.1)), and we work with the following normalized variables:

$$
r:=\frac{\varrho}{R}, \quad \theta:=\frac{\Theta}{\Theta_{\mathrm{y}}}, \quad \gamma:=\frac{\gamma^{\mathrm{p}}}{\gamma_{\mathrm{y}}}, \quad \text { where } \quad \gamma_{\mathrm{y}}:=\frac{k}{\mu}, \quad \Theta_{\mathrm{y}}:=\frac{\gamma_{\mathrm{y}}}{R} .
$$

The constants $\gamma_{\mathrm{y}}$ and $\Theta_{\mathrm{y}}$ are the yield shear and the yield twist, respectively.

We show in section 3 that, under the ansatz (1.10), the flow rule (1.8) reduces to a partial differential inclusion in one dimension that, in terms of the normalized variables (1.13), reads as

$$
\lambda^{2}\left(\frac{\partial^{2} \gamma}{\partial r^{2}}+\frac{1}{r} \frac{\partial \gamma}{\partial r}-\frac{1}{r^{2}} \gamma\right)-\gamma+\theta r \in \partial\left|\frac{\partial \gamma}{\partial \theta}\right| \quad \text { in }(0,1) \times(0, \infty),
$$

where

$$
\partial|s|:= \begin{cases}\{-1\} & \text { if } s<0 \\ {[-1,1]} & \text { if } s=0 \\ \{1\} & \text { if } s>0\end{cases}
$$

The assumption (1.11) and the null microscopic traction at the boundary (see (2.13)) yield

$$
\gamma(r, 0)=0 \quad \text { and } \quad \frac{\partial \gamma}{\partial r}(1, \theta)+\frac{\gamma(1, \theta)}{2}=0 \quad \text { for } \theta \geq 0 \text { and } r \in(0,1) .
$$

1.6. Solution of the torsion problem: $\boldsymbol{L}=0$. To first gain insight into the problem, it is convenient to consider the case $L=0$. Then (1.14a) reduces to

$$
\theta r-\gamma \in \partial\left|\frac{\partial \gamma}{\partial \theta}\right| \quad \text { in } \quad(0, R)
$$

and there is no associated boundary condition. The unique solution of (1.15) with the initial condition $\gamma(r, 0)=0$ is given by

$$
\gamma(r, \theta)=(\theta r-1)_{+},
$$

where $(s)_{+}=\max \{s, 0\}$. From (1.16), two regimes may be identified:

1) an elastic regime, where $\theta \in[0,1]$ and $\gamma=0$;

2 ) an elasto-plastic regime, where $\theta \in(1,+\infty), \gamma=0$ in $[0,1 / \theta]$, and $\gamma>0$ in $(1 / \theta, 1]$.

Thus, an elastic-plastic boundary located at $r=1 / \theta$ separates the region where $\gamma=0$, the so-called elastic core, from the rest of the body, where $\gamma>0$. As $\theta$ increases, the elastic core shrinks, but never disappears.

In terms of normalized variables, (1.12) is best written as $Q=Q_{*} q$, where $Q_{*}:=$ $\frac{2}{3} k \pi R^{3}$ and

$$
q:=3 \int_{0}^{1}(\theta r-\gamma) r^{2} \mathrm{~d} r
$$


is the normalized torque. On substituting (1.16) into (1.17), we obtain, for $\theta \geq 1$,

$$
q=1-\frac{1}{4} \theta^{-3} \text { for all } \theta \geq 1 \text {. }
$$

Notice that $q(\theta) \rightarrow 1$ as $\theta \rightarrow \infty$. Thus, $Q_{*}$ is the ultimate torque that a wire can withstand according to the Levy-Mises theory.

1.7. Solution of the torsion problem: $\boldsymbol{L}>0$. When strain-gradient effects are accounted for, an expression for the torque as simple as (1.18) is not available. In order to get some insight, we need a detailed characterization of the solution of (1.14). In section 4 we note that (1.14a) has a natural formulation in terms of an evolutionary variational inequality (see Definition 4.1), and hence it has a unique solution (see Proposition 4.3). Our main contribution is in section 5, where we show that the unique solution $\gamma$ of (1.14) may be characterized in terms of solutions of suitable boundary-value problems (see Theorem 5.3). As a by-product, our arguments provide an explicit construction of the solution; this construction allows us to identify three regimes:

(1) an initial elastic regime, where $\theta \in[0,1]$ and $\gamma=0$;

(2) an intermediate elasto-plastic regime, where $\theta \in\left[1, \theta_{\lambda}\right), \gamma=0$ in $\left[0, c_{\theta}\right]$, and $\gamma>0$ in $\left(c_{\theta}, 1\right]$

(3) an ultimate plastic regime, where $\theta>\theta_{\lambda}$ and $\gamma>0$ in $(0,1]$.

A relevant feature is apparent from (3): the sample becomes fully plastified when $\theta$ attains a critical twist $\theta_{\lambda}$, in contrast with the case $L=0$, where plastic strain vanishes on $(0,1 / \theta)$.

1.8. Energetic scale effects. The characterization given by Theorem 5.3 allows for an easy computation of the plastic profile and the torque. Numerical results given in Figure 1.1 confirm both the presence of a boundary layer near $\partial \Omega$ and the higher relative strength of thinner wires. In addition, they show the presence of a boundary layer near $c_{\theta}$, the left-endpoint of the plastic region. The characterization given by
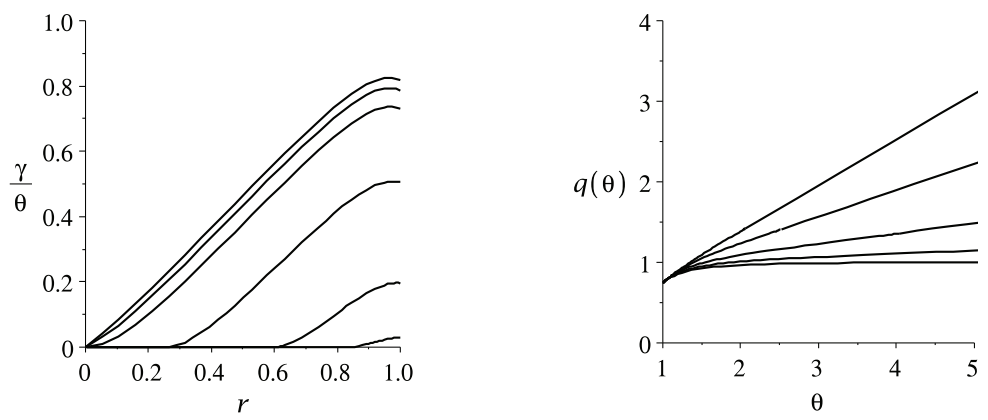

FIG. 1.1. On the left, plots of $\frac{\gamma}{\theta}$ for $\lambda=0.1$ and $\frac{\theta-1}{\theta_{\lambda}-1}=\frac{1}{64}, \frac{1}{16}, \frac{1}{4}, 1,2,4$ (from bottom to top); on the right, plots of normalized torque versus normalized twist for $\lambda=0,0.1,0.2,0.4,0.8$ (from bottom to top).

Theorem 5.3 also allows us to quantify these effects in terms of the (normalized) energetic length-scale $\lambda$. In section 6 , we develop a formal asymptotic expansion as $\lambda \rightarrow 0$ (for fixed $\theta$ ). First we show that

$$
\begin{aligned}
c_{\theta} & \sim \frac{1}{\theta}-\lambda \text { for } \lambda \ll 1 \text { and } \theta \in\left(1, \theta_{\lambda}\right), \\
\gamma & \sim \theta r-1-\frac{1}{2} \lambda(3 \theta-1) e^{-\frac{1-r}{\lambda}} \text { for } \lambda \ll 1,1-r \ll 1, \text { and } \theta>1 .
\end{aligned}
$$


Expansions (1.19) and (1.20) show the appearance of boundary layers of width $O(\lambda)$ near $r=c_{\theta}$ and $r=1$, respectively. Using (1.19) and (1.20), we obtain a scaling law for the critical twist

$$
\theta_{\lambda} \sim \frac{1}{\sqrt{6} \lambda} \text { for } \lambda \ll 1,
$$

and we quantify the higher relative strength of thinner wires by finding the following asymptotic expansion with respect to $\lambda$ of the torque needed to attain a fixed twist $\theta>1$ :

$$
q(\theta) \sim\left\{\begin{array}{lll}
1-\frac{1}{4 \theta^{3}}+\frac{3 \lambda^{2}}{2 \theta}+\frac{9}{2} \lambda^{2}(\theta-1) & \text { for } \lambda \ll 1 & \text { if } 1<\theta<\frac{1}{\sqrt{6} \lambda}, \\
1+\frac{9}{2} \lambda^{2}(\theta-1) & \text { for } \lambda \ll 1 & \text { if } \theta>\frac{1}{\sqrt{6} \lambda} .
\end{array}\right.
$$

Comparing (1.22) with (1.18) and returning to the original variables, we see, in particular, that $Q / Q_{*}$ is proportional to $(L / R)^{2}$. We remark that the theory under scrutiny does not predict any ultimate torque: we conjecture that a defect energy density with linear growth, as deduced in [15], may recover such a feature.

1.9. Hardening versus strengthening scale effects. Our analysis confirms that the normalized energetic length-scale $\lambda$ results in size-dependent strain-hardening: for thinner wires, a higher torque is needed to attain the same twist in the plastic range; that is, the torque-twist curves increase with $\lambda$. However, the experiments reported [9] show an additional size effect: thinner wires can withstand a higher torque without undergoing plastic flow; that is, the torque needed to induce a nonzero plastic shear increases as $R$ decreases. This effect, which goes by the name of size-dependent strengthening, is not displayed by the solutions we construct (regardless of $\lambda$, plastic flow starts at $\theta=1$ and $q(1)=3 / 4$ ). As pointed out in (c) of section 1.4, sizedependent strengthening is expected to be captured by the Gurtin-Anand theory through a nonzero dissipative length-scale $\ell$. In this respect, we mention a recent work by Idiart and Fleck [24], where the torsion problem is considered within the setting of a different flow theory, purely dissipative in nature, derived by Fleck and Willis in [14] (elaborating upon a previous paper by Fleck and Hutchinson [12]; see also related remarks by Anand and Gurtin in [18]). In the so-called "rigid-plastic approximation," Idiart and Fleck obtain an explicit solution which indeed displays size-dependent strengthening effects (see Figure 3(c) of [24]), a result that substantiates the role of dissipative length-scales in modeling this phenomenon.

1.10. Nonsymmetric plastic distortion. The identification of $\operatorname{curl}^{\mathrm{p}}$ as the macroscopic counterpart of the Burgers vector hinges on the assumption that, in the decomposition $\nabla \mathbf{u}=\mathbf{H}^{\mathrm{e}}+\mathbf{H}^{\mathrm{p}}$, the plastic distortion $\mathbf{H}^{\mathrm{p}}$ is symmetric. If this assumption is dropped, then $\mathbf{H}^{\mathrm{p}}=\mathbf{E}^{\mathrm{p}}+\mathbf{W}^{\mathrm{p}}$, with $\mathbf{E}^{\mathrm{p}}$ symmetric and $\mathbf{W}^{\mathrm{p}}$ skewsymmetric. Thus, the additional kinematical unknown $\mathbf{W}^{\mathrm{p}}(x, t) \in \mathbb{R}_{\text {skw }}^{3 \times 3}$, the so-called plastic spin, enters the theory [20] (see also [26, 27, 29] for a related gradient plasticity model dealing with both infinitesimal and finite strains). As pointed out in [21], the ensuing flow rule is then much more complicated; not surprisingly, well-posedness has not been established for such model, unless one includes appropriate hardening terms [10], or restricts attention to particular symmetries [6]. One of the main difficulties lies in controlling $\mathbf{H}^{\mathrm{p}}$ through curl $\mathbf{H}^{\mathrm{p}}$; see [28] for recent results in this direction. In section 7 we show that the trivial generalization of $(1.10)$ with $\mathbf{W}^{\mathrm{p}}=0$ provides a special solution also to the flow rule proposed in [20], where $\psi_{\mathrm{d}}=\frac{1}{2} \mu L^{2} \mid \operatorname{curl}_{\mathbf{H}} \mathbf{H}^{2}$ is postulated. At first glance, this might be taken as an indication that plastic rotations 
do not affect the outcome of a torsion experiment. Instead, we believe that such special solution is nongeneric and unstable, in the sense that nonsymmetric perturbations of the initial configuration will grow with time. It would be interesting to investigate the existence of nonsymmetric special solutions to the flow rule proposed in [20].

\section{Problem setup.}

2.1. Preliminaries. Given $x \in \mathbb{R}^{3}$, we let $x=(\varrho \cos \phi, \varrho \sin \phi, z)$ with $(\varrho, \phi, z) \in$ $(0,+\infty) \times[0,2 \pi) \times \mathbb{R}$, and we introduce the local frame-field vectors

$$
\mathbf{e}_{\langle 1\rangle}(\phi)=(\cos \phi, \sin \phi, 0), \quad \mathbf{e}_{\langle 2\rangle}(\phi)=(-\sin \phi, \cos \phi, 0), \quad \mathbf{e}_{\langle 3\rangle}=(0,0,1) .
$$

It follows from (2.1) that

$$
\mathbf{e}_{\langle i\rangle} \cdot \mathbf{e}_{\langle j\rangle}=\delta_{i j} \quad \text { and } \quad \frac{\partial \mathbf{e}_{\langle i\rangle}}{\partial \phi} \cdot \mathbf{e}_{\langle j\rangle}=\varepsilon_{i j 3},
$$

where $\delta_{i j}$ and $\varepsilon_{i j k}$ are, respectively, the Kroenecker and Levi-Civita symbols. We denote components in the frame-field (2.1) as follows:

$$
(\mathbf{a})_{\langle i\rangle}=\mathbf{a} \cdot \mathbf{e}_{\langle i\rangle}, \quad(\mathbf{A})_{\langle i j\rangle}=\mathbf{A}: \mathbf{e}_{\langle i\rangle} \otimes \mathbf{e}_{\langle j\rangle}, \quad(\mathbb{A})_{\langle i j k\rangle}=\mathbf{A}: \mathbf{e}_{\langle i\rangle} \otimes \mathbf{e}_{\langle j\rangle} \otimes \mathbf{e}_{\langle k\rangle} .
$$

Although these components differ, in general, from those in the standard basis, we still have the usual representation formulas

$$
\mathbf{a}=(\mathbf{a})_{\langle i\rangle} \mathbf{e}_{\langle i\rangle}, \quad \mathbf{A}=(\mathbf{A})_{\langle i j\rangle} \mathbf{e}_{\langle i\rangle} \otimes \mathbf{e}_{\langle j\rangle}, \quad \mathbb{A}=(\mathbb{A})_{\langle i j k\rangle} \mathbf{e}_{\langle i\rangle} \otimes \mathbf{e}_{\langle j\rangle} \otimes \mathbf{e}_{\langle k\rangle},
$$

along with the usual componentwise multiplication rules:

$$
(\mathbf{A a})_{\langle i\rangle}=(\mathbf{A})_{\langle i j\rangle}(\mathbf{a})_{\langle j\rangle} \quad \text { and } \quad(\mathbb{A} \mathbf{a})_{\langle i j\rangle}=(\mathbb{A})_{\langle i j k\rangle}(\mathbf{a})_{\langle k\rangle} .
$$

Given a tensor field $\mathbf{A}$, we define curlA (see [19, p. 11]) as the unique tensor field such that $(\operatorname{curl} \mathbf{A}) \mathbf{a}=\operatorname{curl}\left(\mathbf{A}^{T} \mathbf{a}\right)$ for every constant vector field $\mathbf{a}$ (we warn the reader that another definition of the curl operator, which does not involve transposition, may be found in the literature; see, for instance, [27]). Using local components, we have

$$
(\operatorname{curl} \mathbf{A})_{\langle i j\rangle}=\varepsilon_{i k l}(\nabla \mathbf{A})_{\langle j l k\rangle} \text {. }
$$

If $\mathbf{A}$ is symmetric, then the following identity holds [19, eq. (13)]:

$$
\text { curlcurl } \mathbf{A}=-\Delta \mathbf{A}+2 \operatorname{sym} \nabla \operatorname{div} \mathbf{A}-\nabla \nabla \operatorname{tr} \mathbf{A}+(\Delta \operatorname{tr} \mathbf{A}-\operatorname{div} \operatorname{div} \mathbf{A}) \mathbf{I} .
$$

We next recall that

$(\mathbf{a} \otimes \mathbf{b}) \mathbf{c}=(\mathbf{b} \cdot \mathbf{c}) \mathbf{a}, \quad \operatorname{sym}(\mathbf{a} \otimes \mathbf{b})=\frac{1}{2}(\mathbf{a} \otimes \mathbf{b}+\mathbf{b} \otimes \mathbf{a}), \quad$ and $\quad(\mathbf{a} \otimes \mathbf{b})_{0}=\mathbf{a} \otimes \mathbf{b}-\frac{1}{3}(\mathbf{a} \cdot \mathbf{b}) \mathbf{I}$, and we summarize some useful rules of tensor calculus, to be used later: given a vector field $\mathbf{a}(\varrho, \theta, z)$, its gradient $\nabla \mathbf{a}$ can be represented as

$$
\nabla \mathbf{a}=\frac{\partial \mathbf{a}}{\partial \varrho} \otimes \mathbf{e}_{\langle 1\rangle}+\frac{1}{\varrho} \frac{\partial \mathbf{a}}{\partial \phi} \otimes \mathbf{e}_{\langle 2\rangle}+\frac{\partial \mathbf{a}}{\partial z} \otimes \mathbf{e}_{\langle 3\rangle} ;
$$

a similar formula holds for a tensor field $\mathbf{A}(\varrho, \theta, z)$; moreover,

$$
\operatorname{div} \mathbf{A}=\frac{\partial \mathbf{A}}{\partial \varrho} \mathbf{e}_{\langle 1\rangle}+\frac{1}{\varrho} \frac{\partial \mathbf{A}}{\partial \phi} \mathbf{e}_{\langle 2\rangle}+\frac{\partial \mathbf{A}}{\partial z} \mathbf{e}_{\langle 3\rangle} .
$$

In view of (2.8), (2.2), and (2.10), the following implication is easily established:

$$
\mathbf{A}(\varrho, \phi)=\alpha(\varrho) \operatorname{sym}\left(\mathbf{e}_{\langle 2\rangle}(\phi) \otimes \mathbf{e}_{\langle 3\rangle}\right) \Rightarrow \operatorname{div} \mathbf{A}=\mathbf{0} .
$$


2.2. The $3 \mathrm{D}$ problem. We now specialize the theory to the cylinder $\Omega_{R}$ described by (1.9). The torque sustained by the cylinder is, by definition,

$$
Q:=\mathbf{e}_{\langle 3\rangle} \cdot \int_{\Sigma} \varrho \mathbf{e}_{\langle 1\rangle} \times \mathbf{T} \mathbf{e}_{\langle 3\rangle} \mathrm{d} S,
$$

where $\Sigma$ is any cross section of $\Omega_{R}$ (for instance, $\Sigma=\Omega_{R} \cap\{z=0\}$ will do). We neglect inertia and other body forces, and we require the lateral side of the cylinder to be traction-free:

$$
\mathbf{T n}=0 \quad \text { and } \quad \mathbb{K}^{\mathrm{p}} \mathbf{n}=0 \quad \text { on } \partial \Omega_{R},
$$

where $\mathbf{n}$ is the outward unit normal to $\partial \Omega_{R}$. It follows from $(1.2 \mathrm{~b})$ that

$$
\mathbf{T}=2 \mu \mathbf{E}_{0}^{\mathrm{e}}+\kappa \operatorname{tr}\left(\mathbf{E}^{\mathrm{e}}\right) \mathbf{I}
$$

In view of (1.6), the constitutive equations $(1.5 \mathrm{~b})$ reduce to

$$
\mathbf{T}^{\mathrm{p}} \in \partial \delta\left(\dot{\mathbf{E}}^{\mathrm{p}}\right) \quad \text { and } \quad \mathbb{K}^{\mathrm{p}}=\frac{\partial \psi_{d}\left(\nabla \mathbf{E}^{\mathrm{p}}\right)}{\partial \nabla \mathbf{E}^{\mathrm{p}}} .
$$

When worked out in components with the aid of (2.6), the constitutive equation $(2.15)_{2}$ turns into

$$
\begin{aligned}
& \left(\mathbb{K}^{\mathrm{p}}\right)_{\langle j q p\rangle}=\mu L^{2}\left[\left(\nabla \mathbf{E}^{\mathrm{p}}\right)_{\langle j q p\rangle}-\frac{1}{2}\left(\left(\nabla \mathbf{E}^{\mathrm{p}}\right)_{\langle j p q\rangle}+\left(\nabla \mathbf{E}^{\mathrm{p}}\right)_{\langle q p j\rangle}\right)\right. \\
& \left.+\frac{1}{3}(1+\eta) \delta_{j q}\left(\nabla \mathbf{E}^{\mathrm{p}}\right)_{\langle r p r\rangle}-\frac{\eta}{2}\left(\varepsilon_{i p q} \varepsilon_{j r s}+\varepsilon_{i p j} \varepsilon_{q r s}\right)(\nabla \mathbf{E})_{\langle i s r\rangle}\right]
\end{aligned}
$$

(cf. [22, eq. (90.47)]), so that

$$
\operatorname{div} \mathbb{K}^{\mathrm{p}}=\mu L^{2}\left(\Delta \mathbf{E}^{\mathrm{p}}-\operatorname{sym}\left(\nabla \operatorname{div} \mathbf{E}^{\mathrm{p}}\right)+\frac{1}{3}(1+\eta)\left(\operatorname{div} \operatorname{div} \mathbf{E}^{\mathrm{p}}\right) \mathbf{I}+\eta \operatorname{curlcurl} \mathbf{E}^{\mathrm{p}}\right)
$$

(see also [22, eq. (90.64)], with $\lambda=\eta$, which, however, contains a typo). Substituting (2.17) into (1.5a) we obtain (1.8). Combining (1.2c), (1.5a), (2.15), (2.13), and imposing a null initial plastic strain, we see that the system governing the evolution of displacement $\mathbf{u}(x, t)$ and plastic strain $\mathbf{E}^{\mathrm{p}}(x, t)$ in the cylinder $\Omega_{R}$ is

$$
\begin{cases}\operatorname{div} \mathbf{T}=\mathbf{0} & \text { in } \Omega_{R} \times(0,+\infty), \\ \operatorname{div} \mathbb{K}^{\mathrm{p}}+\mathbf{T}_{0} \in \partial \delta\left(\dot{\mathbf{E}}^{\mathrm{p}}\right) & \text { in } \Omega_{R} \times(0,+\infty), \\ \mathbf{T} \mathbf{n}=0 & \text { on } \partial \Omega_{R} \times(0,+\infty), \\ \mathbb{K}^{\mathrm{p}} \mathbf{n}=0 & \text { on } \partial \Omega_{R} \times(0,+\infty), \\ \mathbf{E}^{\mathrm{p}}(\cdot, 0)=0 & \text { in } \Omega_{R},\end{cases}
$$

where stress $\mathbf{T}(x, t) \in \mathbb{R}_{\text {sym }}^{3 \times 3}$ and polar microstress $\mathbb{K}^{\mathrm{p}}(x, t) \in \mathbb{R}_{0}^{3 \times 3 \times 3}$ are related to displacement gradient and plastic strain through (2.14) and (2.16).

3. The torsion problem. We now argue that the ansatz (1.10)-(1.11) yields a special class of solutions of the bulk system (2.18). As explained in the introduction, we replace $t$ with $\Theta$ as the independent variable: we henceforth maintain that a superimposed dot denotes partial differentiation with respect to $\Theta$. In place of (1.10), we then write

$$
\begin{aligned}
& \mathbf{u}(\varrho, \phi, z, \Theta)=z \Theta \varrho \mathbf{e}_{\langle 2\rangle}(\phi), \\
& \mathbf{E}^{\mathrm{p}}(\varrho, \phi, \Theta)=\gamma^{\mathrm{p}}(\varrho, \Theta) \operatorname{sym}\left(\mathbf{e}_{\langle 2\rangle}(\phi) \otimes \mathbf{e}_{\langle 3\rangle}\right) .
\end{aligned}
$$


Our first task is to verify that the stress $\mathbf{T}$ resulting from (3.1) satisfies $(2.18)_{1}$ and $(2.18)_{3}$. To begin, we use (2.2) and (2.9) to obtain $\nabla \mathbf{u}=z \Theta \mathbf{e}_{\langle 2\rangle} \otimes \mathbf{e}_{\langle 1\rangle}-z \Theta \mathbf{e}_{\langle 1\rangle} \otimes$ $\mathbf{e}_{\langle 2\rangle}+\varrho \Theta \mathbf{e}_{\langle 2\rangle} \otimes \mathbf{e}_{\langle 3\rangle}$, whence

$$
\operatorname{sym} \nabla \mathbf{u}=\varrho \Theta \operatorname{sym}\left(\mathbf{e}_{\langle 2\rangle} \otimes \mathbf{e}_{\langle 3\rangle}\right) .
$$

By combining (1.2a) with (3.1b) and (3.2), we get

$$
\mathbf{E}^{\mathrm{e}}=\left(\varrho \Theta-\gamma^{\mathrm{p}}\right) \operatorname{sym}\left(\mathbf{e}_{\langle 2\rangle} \otimes \mathbf{e}_{\langle 3\rangle}\right) .
$$

On substituting (3.3) in (2.14), since $\operatorname{tr} \mathbf{E}^{\mathrm{e}}=0$ we find

$$
\mathbf{T}=2 \mu\left(\Theta \varrho-\gamma^{\mathrm{p}}\right) \operatorname{sym}\left(\mathbf{e}_{\langle 2\rangle} \otimes \mathbf{e}_{\langle 3\rangle}\right) .
$$

From (2.11) and (3.4) we conclude that (2.18) $)_{1}$ is satisfied. Furthermore, since $\mathbf{n}=$ $\mathbf{e}_{\langle 1\rangle}$ on $\partial \Omega_{R}$, and since $\operatorname{sym}\left(\mathbf{e}_{\langle 2\rangle} \otimes \mathbf{e}_{\langle 3\rangle}\right) \mathbf{e}_{\langle 1\rangle}=0$ by $(2.8)_{1,2}$, we conclude that $(2.18)_{3}$ holds true.

Our next task it to show that, under $(3.1),(2.18)_{2}$ is translated into

$$
\mu\left(\Theta \varrho-\gamma^{\mathrm{p}}\right)+\mu(1-\eta) \frac{L^{2}}{2}\left(\frac{\partial^{2} \gamma^{\mathrm{p}}}{\partial \varrho^{2}}+\frac{1}{\varrho} \frac{\partial \gamma^{\mathrm{p}}}{\partial \varrho^{2}}-\frac{\gamma^{\mathrm{p}}}{\varrho^{2}}\right) \in k \partial\left|\dot{\gamma}^{\mathrm{p}}\right| \quad \text { in } \quad(0, R),
$$

and that the initial condition $(2.18)_{5}$ and the null-microtraction condition $(2.18)_{4}$ are translated into

$$
\gamma^{\mathrm{p}}(\varrho, 0)=0 \quad \text { and } \quad \frac{\partial \gamma^{\mathrm{p}}}{\partial \varrho}(R, \Theta)+\frac{1}{2} \gamma^{\mathrm{p}}(R, \Theta)=0 .
$$

First, we observe that, by (2.11), $\mathbf{E}^{\mathrm{p}}$ has null divergence:

$$
\operatorname{div} \mathbf{E}^{\mathrm{p}}=\mathbf{0}
$$

Thus, since $\operatorname{tr} \mathbf{E}^{\mathrm{p}}=0$, the identity (2.7) yields curlcurl| $\mathbf{E}^{\mathrm{p}}=-\Delta \mathbf{E}^{\mathrm{p}}$. Hence, on recalling $(2.17)$, we see that $(2.18)_{2}$ reduces to

$$
\mathbf{T}_{0}+\mu(1-\eta) L^{2} \Delta \mathbf{E}^{\mathrm{p}} \in \partial \delta\left(\dot{\mathbf{E}}^{\mathrm{p}}\right) .
$$

Next, using the tensorial version of (2.9) and (2.2), we find from (3.1b) that

$$
\nabla \mathbf{E}^{\mathrm{p}}=\frac{\partial \gamma^{\mathrm{p}}}{\partial \varrho} \operatorname{sym}\left(\mathbf{e}_{\langle 2\rangle} \otimes \mathbf{e}_{\langle 3\rangle}\right) \otimes \mathbf{e}_{\langle 1\rangle}-\varrho^{-1} \gamma^{\mathrm{p}} \operatorname{sym}\left(\mathbf{e}_{\langle 1\rangle} \otimes \mathbf{e}_{\langle 3\rangle}\right) \otimes \mathbf{e}_{\langle 2\rangle} .
$$

Then, using the identity (2.10) with $\mathbf{A}=\nabla \mathbf{E}^{\mathrm{p}}$ we arrive at

$$
\Delta \mathbf{E}^{\mathrm{p}}=\operatorname{div} \nabla \mathbf{E}^{\mathrm{p}}=\left(\frac{\partial^{2} \gamma^{\mathrm{p}}}{\partial \varrho^{2}}+\frac{1}{\varrho} \frac{\partial \gamma^{\mathrm{p}}}{\partial \varrho^{2}}-\frac{\gamma^{\mathrm{p}}}{\varrho^{2}}\right) \operatorname{sym}\left(\mathbf{e}_{\langle 2\rangle} \otimes \mathbf{e}_{\langle 3\rangle}\right) .
$$

From (3.4), taking into account $(2.8)_{2.3}$ and $(2.2)_{1}$, we see that $\mathbf{T}_{0}=\mathbf{T}$. Hence, plugging (3.4) and (3.10) into (3.8), we obtain that the inclusion $(2.18)_{2}$ is equivalent to

$$
\begin{aligned}
& 2 \mu\left[\left(\Theta \varrho-\gamma^{\mathrm{p}}\right)+(1-\eta) \frac{L^{2}}{2}\left(\frac{\partial^{2} \gamma^{\mathrm{p}}}{\partial \varrho^{2}}+\frac{1}{\varrho} \frac{\partial \gamma^{\mathrm{p}}}{\partial \varrho^{2}}-\frac{\gamma^{\mathrm{p}}}{\varrho^{2}}\right)\right] \operatorname{sym}\left(\mathbf{e}_{\langle 2\rangle} \otimes \mathbf{e}_{\langle 3\rangle}\right) \\
& \quad \in \partial \delta\left(\dot{\gamma}^{\mathrm{p}} \operatorname{sym}\left(\mathbf{e}_{\langle 2\rangle} \otimes \mathbf{e}_{\langle 3\rangle}\right)\right)
\end{aligned}
$$


granted the ansatz (3.1). Now, denoting by $\alpha$ and $\beta$ any pair of scalars, and by $\mathbf{A} \neq \mathbf{0}$ a second-order tensor, we have

$$
\alpha \mathbf{A} \in \partial \delta(\beta \mathbf{A}) \Longleftrightarrow \alpha \in \frac{\sqrt{2} k}{|\mathbf{A}|} \partial|\beta| .
$$

Since $\left|\operatorname{sym}\left(\mathbf{e}_{\langle 2\rangle} \otimes \mathbf{e}_{\langle 3\rangle}\right)\right|=\frac{1}{\sqrt{2}}$, (3.12) implies that (3.5) is equivalent to (3.11), and hence to $(2.18)_{2}$. Finally, we consider the null microtraction condition $(2.18)_{4}$. Since $\mathbf{n}=\mathbf{e}_{\langle 1\rangle}$, from $(2.2)_{1},(2.3)$, and $(2.5)$ we have $\left(\mathbb{K}^{\mathrm{p}} \mathbf{n}\right)_{\langle j q\rangle}=\left(\mathbb{K}^{\mathrm{p}}\right)_{\langle j q p\rangle}(\mathbf{n})_{\langle p\rangle}=$ $\left(\mathbb{K}^{\mathrm{p}}\right)_{\langle j q 1\rangle}$. Thus, by (2.4), $\mathbb{K}^{\mathrm{P}} \mathbf{n}=\left(\mathbb{K}^{\mathrm{P}} \mathbf{n}\right)_{\langle j q\rangle} \mathbf{e}_{\langle j\rangle} \otimes \mathbf{e}_{\langle q\rangle}=\left(\mathbb{K}^{\mathrm{p}}\right)_{\langle j q 1\rangle} \mathbf{e}_{\langle j\rangle} \otimes \mathbf{e}_{\langle q\rangle}$. By working out (3.9) and (2.16), it turns out that all components $\left(\mathbb{K}^{\mathrm{P}}\right)_{\langle j q 1\rangle}$ vanish, except for $\left(\mathbb{K}^{\mathrm{p}}\right)_{\langle 231\rangle}=\left(\mathbb{K}^{\mathrm{p}}\right)_{\langle 321\rangle}=\frac{\mu L^{2}}{2}(1-\eta)\left(\frac{\partial \gamma^{\mathrm{p}}}{\partial \varrho}+\frac{1}{2} \gamma^{\mathrm{p}}\right)$. Therefore, we conclude that

$$
\mathbb{K}^{\mathrm{p}} \mathbf{n}=\mu L^{2}(1-\eta)\left(\frac{\partial \gamma^{\mathrm{p}}}{\partial \varrho}+\frac{1}{2} \gamma^{\mathrm{p}}\right) \operatorname{sym}\left(\mathbf{e}_{\langle 2\rangle} \otimes \mathbf{e}_{\langle 3\rangle}\right),
$$

and hence $(2.18)_{4}$ yields $(3.6)_{2}$. Finally, $(3.6)_{1}$ follows immediately from $(2.18)_{5}$.

By (1.2b) and (3.3), and since $\left|\operatorname{sym}\left(\mathbf{e}_{\langle 2\rangle} \otimes \mathbf{e}_{\langle 3\rangle}\right)\right|^{2}=\frac{1}{2}$, the elastic-energy density is $\psi_{\mathrm{e}}=\mu \mathbf{E}^{\mathrm{e}}: \mathbf{E}^{\mathrm{e}}=\frac{\mu}{2}\left(\varrho \Theta-\gamma^{\mathrm{p}}\right)^{2}$. Moreover, using (3.9) and (2.6), we find that $\left(\operatorname{curl} \mathbf{E}^{\mathrm{p}}\right)_{\langle i j\rangle}=0$ if $i \neq j$ and

$$
\left(\operatorname{curl} \mathbf{E}^{\mathrm{p}}\right)_{\langle 11\rangle}=-\frac{1}{2} \frac{\gamma^{\mathrm{p}}}{\varrho}, \quad\left(\operatorname{curl} \mathbf{E}^{\mathrm{p}}\right)_{\langle 22\rangle}=-\frac{1}{2} \frac{\partial \gamma^{\mathrm{p}}}{\partial \varrho}, \quad\left(\operatorname{curl} \mathbf{E}^{\mathrm{p}}\right)_{\langle 33\rangle}=\frac{1}{2}\left(\frac{\partial \gamma^{\mathrm{p}}}{\partial \varrho}+\frac{\gamma^{\mathrm{p}}}{\varrho}\right) .
$$

Thus, by (1.4) the defect-energy density is

$$
\psi_{\mathrm{d}}=\mu \frac{L^{2}}{4}(1-\eta)\left[\left(\frac{\partial \gamma^{\mathrm{p}}}{\partial \varrho}\right)^{2}+\frac{\gamma}{\varrho} \frac{\partial \gamma^{\mathrm{p}}}{\partial \varrho}+\left(\frac{\gamma^{\mathrm{p}}}{\varrho}\right)^{2}\right] .
$$

By integrating the free-energy density $\psi=\psi_{\mathrm{e}}+\psi_{\mathrm{d}}$ over any cross section of $\Omega_{R}$, we obtain the free energy per unit length along the cylinder axis:

$\mathcal{F}\left(\gamma^{\mathrm{p}}, \Theta\right)=2 \pi \mu \int_{0}^{R} \frac{1}{2}\left\{\left(\Theta \varrho-\gamma^{\mathrm{p}}\right)^{2}+(1-\eta) \frac{L^{2}}{2}\left[\left(\frac{\partial \gamma^{\mathrm{p}}}{\partial \varrho}\right)^{2}+\frac{\gamma^{\mathrm{p}}}{\varrho} \frac{\partial \gamma^{\mathrm{p}}}{\partial \varrho}+\left(\frac{\gamma^{\mathrm{p}}}{\varrho}\right)^{2}\right]\right\} \varrho \mathrm{d} \varrho$.

By $(3.1), \delta\left(\dot{\mathbf{E}}^{\mathrm{p}}\right)=\sqrt{2} k\left|\operatorname{sym}\left(\mathbf{e}_{\langle 1\rangle} \otimes \mathbf{e}_{\langle 2\rangle}\right)\right|\left|\dot{\gamma}^{\mathrm{p}}\right|=k\left|\dot{\gamma}^{\mathrm{p}}\right|$. Again, integration over any cross section of $\Omega_{R}$ yields the dissipation rate per unit length along the axis:

$$
\mathcal{D}\left(\dot{\gamma}^{\mathrm{p}}\right)=2 \pi k \int_{0}^{R}\left|\dot{\gamma}^{\mathrm{p}}\right| \varrho \mathrm{d} \varrho .
$$

\section{Formulation and solution to the torsion problem.}

4.1. Normalization. We pass to the normalized variables (1.13), we introduce the normalized length-scale:

$$
\lambda:=\sqrt{\frac{(1-\eta)}{2}} \frac{L}{R}
$$

and we define the linear operator

$$
\mathcal{L} \gamma:=\lambda^{2}\left(\gamma^{\prime \prime}+\frac{\gamma^{\prime}}{r}-\frac{\gamma}{r^{2}}\right)-\gamma
$$


By virtue of (1.13), the partial differential inclusion (3.5) and the conditions (3.6) are equivalent to (1.14), which we rewrite for convenience:

$$
\begin{cases}\mathcal{L} \gamma+\theta r \in \partial|\dot{\gamma}| & \text { in } \quad(0,1) \times(0,+\infty), \\ \gamma^{\prime}(1, \theta)+\frac{1}{2} \gamma(1, \theta)=0 & \text { for } \theta>0, \\ \gamma(r, 0)=0 & \text { for } r \in(0,1),\end{cases}
$$

where now $\dot{\gamma}=\frac{\partial \gamma}{\partial \theta}$. In terms of the normalized variables the torque (1.12) can be written as in (1.17), and free energy and dissipation-rate are expressed, respectively, by $\mathcal{F}\left(\gamma^{\mathrm{p}}, \Theta\right)=\mathcal{E}_{*} \mathscr{F}(\gamma, \theta)$ and $\mathcal{D}\left(\gamma^{\mathrm{p}}, \Theta\right)=\mathcal{E}_{*} \mathscr{D}(\gamma, \theta)$, where $\mathcal{E}_{*}:=2 \pi R^{2} \frac{k^{2}}{\mu}$ and

$\mathscr{F}(\gamma, \theta):=\int_{0}^{1} \frac{1}{2}\left((\theta r-\gamma)^{2}+\lambda^{2}\left(\gamma^{\prime 2}+\frac{\gamma^{\prime} \gamma}{r}+\left(\frac{\gamma}{r}\right)^{2}\right)\right) r \mathrm{~d} r, \quad \mathscr{D}(\dot{\gamma}):=\int_{0}^{1}|\dot{\gamma}| r \mathrm{~d} r$.

We'll find it more convenient to work with the effective energy

$$
\mathscr{E}(\gamma, \theta):=\mathscr{F}(\gamma, \theta)-\frac{\theta^{2}}{8}=\frac{1}{2} \int_{0}^{1}\left(\gamma^{2}+\lambda^{2}\left(\gamma^{\prime 2}+\frac{\gamma^{\prime} \gamma}{r}+\left(\frac{\gamma}{r}\right)^{2}\right)\right) r \mathrm{~d} r-\theta \int_{0}^{1} \gamma r^{2} \mathrm{~d} r .
$$

4.2. The evolutionary variational inequality. The structure of $\mathscr{E}$ suggests that the natural functional setting for (4.3) is the space

$$
H:=\overline{C_{c}^{\infty}((0,1])}\|\cdot\|_{r}, \quad \text { where } \quad\|g\|_{r}:=\int_{0}^{1}\left(g^{\prime 2}+\left(\frac{g}{r}\right)^{2}\right) r \mathrm{~d} r .
$$

We first notice that

$$
\sup _{(0,1)}|\gamma|^{2} \leq\|\gamma\|_{H}^{2} \quad \text { and } \quad \lim _{r \rightarrow 0^{+}} \gamma(r)=0 \quad \text { for all } \quad \gamma \in H .
$$

Indeed, if $\|\gamma\|_{H}$ is finite, then a sequence $r_{n} \rightarrow 0^{+}$exists such that $\gamma^{2}\left(r_{n}\right) \rightarrow 0$. For any $r>r_{n}$,

$$
\left|\gamma^{2}(r)-\gamma^{2}\left(r_{n}\right)\right|=\left|2 \int_{r_{n}}^{r} \gamma \gamma^{\prime} \mathrm{d} r\right| \leq\left(\int_{0}^{r} r\left(\gamma^{\prime}\right)^{2} \mathrm{~d} r\right)^{1 / 2}\left(\int_{0}^{r} \frac{\gamma^{2}}{r} \mathrm{~d} r\right)^{1 / 2} \lesssim\|\gamma\|_{H}^{2} .
$$

Taking the limit as $n \rightarrow+\infty$ yields (4.4) 1 . A further passage to the limit as $r \rightarrow 0^{+}$ delivers (4.4) $)_{2}$. A simple computation shows that $D_{\gamma} \mathscr{E}(\gamma, \theta) \in H^{\prime}$ is given by

$$
\left\langle D_{\gamma} \mathscr{E}(\gamma, \theta), \tilde{\gamma}\right\rangle=a(\gamma, \tilde{\gamma})-\langle\ell(\theta), \tilde{\gamma}\rangle,
$$

where $\langle\cdot, \cdot\rangle$ denotes the pairing between $H^{\prime}$ and $H, a: H \times H \rightarrow \mathbb{R}$ is the symmetric bilinear form

$$
a(\gamma, \tilde{\gamma}):=\int_{0}^{1}\left(\gamma \tilde{\gamma}+\lambda^{2}\left(\gamma^{\prime} \tilde{\gamma}^{\prime}+\frac{\gamma^{\prime} \tilde{\gamma}+\tilde{\gamma}^{\prime} \gamma}{2 r}+\frac{\tilde{\gamma} \gamma}{r^{2}}\right)\right) r \mathrm{~d} r
$$

and $\ell(\theta): H \rightarrow \mathbb{R}$ is the linear form

$$
\langle\ell(\theta), \gamma\rangle:=\theta \int_{0}^{1} r^{2} \gamma \mathrm{d} r
$$


On the other hand, a formal integration by parts shows that if $\gamma$ is a smooth solution to $(4.3)$, then

$$
\left\langle D_{\gamma} \mathscr{E}(\gamma, \theta), \tilde{\gamma}\right\rangle=-\int_{0}^{1}(\mathcal{L} \gamma+\theta r) \tilde{\gamma} r \mathrm{~d} r
$$

This suggests writing (4.3) in its subdifferential formulation:

$$
\partial \mathscr{D}(\dot{\gamma})+D_{\gamma} \mathscr{E}(\gamma, \theta) \ni 0
$$

where $\partial \mathscr{D}$ is defined by

$$
\xi \in \partial \mathscr{D}(\dot{\gamma}) \Longleftrightarrow\langle-\xi, \widetilde{\gamma}-\dot{\gamma}\rangle \geq \mathscr{D}(\dot{\gamma})-\mathscr{D}(\widetilde{\gamma}) \text { for all } \widetilde{\gamma} \in H
$$

We recognize in (4.5) and (4.8) the standard format of an evolutionary variational inequality.

Definition 4.1. Let $\gamma \in W_{\mathrm{loc}}^{1,1}([0,+\infty) ; H)$. We say that $\gamma$ solves $(4.3)$ if $\gamma(0)=$ 0 and

$$
a(\gamma, \tilde{\gamma}-\dot{\gamma})-\langle\ell(\theta), \tilde{\gamma}-\dot{\gamma}\rangle \geq \mathscr{D}(\dot{\gamma})-\mathscr{D}(\tilde{\gamma}) \quad \text { for all } \tilde{\gamma} \in H \text { and a.e. } \theta>0 .
$$

We observe the following lemma.

Lemma 4.2. The bilinear form $a: H \times H \rightarrow \mathbb{R}$ given by (4.6) is continuous and coercive, and

$$
a(\gamma, \tilde{\gamma})=\int_{0}^{1}\left(\gamma \tilde{\gamma}+\lambda^{2}\left(\gamma^{\prime} \tilde{\gamma}^{\prime}+\frac{\gamma \tilde{\gamma}}{r^{2}}\right)\right) r \mathrm{~d} r+\frac{\lambda^{2}}{2} \gamma(1) \tilde{\gamma}(1) \quad \text { for all } \gamma, \tilde{\gamma} \in H .
$$

Proof. The reformulation (4.10) follows from noting that

$$
\int_{0}^{1}\left(\gamma^{\prime} \tilde{\gamma}+\tilde{\gamma}^{\prime} \gamma\right) \mathrm{d} r \stackrel{(4.4)_{2}}{=} \gamma(1) \tilde{\gamma}(1) \quad \text { for all } \gamma, \tilde{\gamma} \in H .
$$

In view of (4.10), coercivity is immediate, and continuity follows, recalling (4.4) ${ }_{1}$.

According to [23, Theorem 7.3] the Lipschitz continuity of $\mathscr{D}$ with respect to the norm $\|\cdot\|_{H}$ and Lemma 4.2 give existence and uniqueness.

Proposition 4.3. There exists a unique $\gamma \in W_{\mathrm{loc}}^{1,1}([0,+\infty) ; H)$ that solves (4.3) in the sense of Definition 4.1.

5. Characterization of the solution. To characterize the solution to (4.3) we first try to get some hints from the explicit result available in the standard torsion problem, that is, when $\lambda=0$. In terms of normalized variables (1.13), the solution for $\lambda=0$ is given by (1.16), and has the following property: for each $\theta>1$ there exists an elasto-plastic radius $c_{\theta}$ such that

$$
\begin{cases}\dot{\gamma}(r, \theta)=0 \text { and } \gamma(r, \theta)=0 & \text { if } r \in\left[0, c_{\theta}\right), \\ \dot{\gamma}(r, \theta)>0 \text { and } \gamma(r, \theta)>0 & \text { if } r \in\left(c_{\theta}, 1\right] .\end{cases}
$$

Moreover, $c_{\theta}=1$ if $\theta \in[0,1]$. When looking for a solution of (4.3) for $\lambda>0$, it is natural to search first among plastic profiles consistent with (5.1). For all fixed $\theta>1$, any such profile must satisfy

$$
\lambda^{2}\left(\gamma^{\prime \prime}+\frac{1}{r} \gamma^{\prime}-\frac{\gamma}{r^{2}}\right)-\gamma=1-\theta r \quad \text { on }\left(c_{\theta}, 1\right),
$$


along with the boundary condition $(4.3)_{2}$ and the left-end condition

$$
\lim _{r \rightarrow c_{\theta}^{+}} \gamma(r, \theta)=0,
$$

the latter implicit in the choice of $H$ as ambient space if $c_{\theta}=0$. There is, however, an extra condition coming from (4.3) and from $\gamma(\cdot, \theta) \in H$, namely,

$$
\lim _{r \rightarrow c_{\theta}^{+}} \gamma^{\prime}(r, \theta)=0 \text { for } c_{\theta}>0 .
$$

This condition is necessary for $r \mapsto \gamma^{\prime}(\theta, r)$ to be continuous across $c_{\theta}$ : without such continuity, $r \mapsto \gamma^{\prime \prime}(\theta, r)$ would not be square-integrable across $c_{\theta}$, whereas all the other terms in (4.3) are.

By putting together (5.2), (5.3), and (5.4), and the microfree condition $(4.3)_{2}$, we obtain the following free boundary problem:

$$
\left(\mathscr{P}_{\theta}\right)\left\{\begin{array}{l}
\lambda^{2}\left(\gamma^{\prime \prime}+\frac{1}{r} \gamma^{\prime}-\frac{\gamma}{r^{2}}\right)-\gamma=1-\theta r \quad \text { on }\left(c_{\theta}, 1\right), \\
\gamma\left(c_{\theta}\right)=\gamma^{\prime}\left(c_{\theta}\right)=0, \quad \gamma^{\prime}(1)+\frac{1}{2} \gamma(1)=0 .
\end{array}\right.
$$

Here $c_{\theta}$ is an additional unknown to be determined together with $\gamma$ (at variance with the case $\lambda=0$, when $c_{\theta}$ is given by $\left.1 / \theta\right)$. It turns out that $\left(\mathscr{P}_{\theta}\right)$ is well posed up to a critical twist $\theta_{\lambda}$.

Lemma 5.1. Let $\lambda>0$. There exists a critical twist $\theta_{\lambda}>1$ such that for all $\theta \in\left(1, \theta_{\lambda}\right)$, problem $\left(\mathscr{P}_{\theta}\right)$ has a unique solution $\left(c_{\theta}, \gamma_{\theta}\right) \in(0,1) \times C^{\infty}\left(\left[c_{\theta}, 1\right]\right)$, and has no solution for $\theta>\theta_{\lambda}$. Furthermore we have the following:

(i) $c_{\theta}$ is strictly decreasing and uniformly Lipschitz continuous with respect to $\theta$, with $c_{1}=1$ and $c_{\theta_{\lambda}}=0$

(ii) $c_{\theta}<1 / \theta$ for all $\theta \in\left(1, \theta_{\lambda}\right)$;

(iii) $\gamma_{\theta}>0$ in $\left(c_{\theta}, 1\right]$ for all $\theta \in\left(1, \theta_{\lambda}\right)$;

(iv) if $1<\theta_{1}<\theta_{2}<\theta_{\lambda}$, then $\gamma_{\theta_{1}}<\gamma_{\theta_{2}}$ in $\left[c_{\theta_{1}}, 1\right]$.

It follows from part (i) that, as $\theta$ attains the critical twist $\theta_{\lambda}$, the elasto-plastic boundary hits the origin $r=0$. Hence one expects that for $\theta \geq \theta_{\lambda}$ the plastic-shear profile solves

$$
\left(\mathscr{P}_{\theta}^{\prime}\right)\left\{\begin{array}{l}
\lambda^{2}\left(\gamma^{\prime \prime}+\frac{1}{r} \gamma^{\prime}-\frac{\gamma}{r^{2}}\right)-\gamma=1-\theta r \quad \text { on }(0,1) \\
\gamma(0)=0, \quad \gamma^{\prime}(1)+\frac{1}{2} \gamma(1)=0
\end{array}\right.
$$

which is well posed for all $\theta \in \mathbb{R}$. $\left(\mathscr{P}_{\theta}^{\prime}\right)$ :

Lemma 5.2. Let $\lambda>0$. For all $\theta \in \mathbb{R}$ there exists a unique solution $\bar{\gamma}_{\theta} \in H$ of

$$
a\left(\bar{\gamma}_{\theta}, \tilde{\gamma}\right)=\int_{0}^{1}(\theta r-1) \tilde{\gamma} r \mathrm{~d} r \quad \text { for all } \tilde{\gamma} \in H,
$$

with $a(\cdot, \cdot)$ given by (4.6). Furthermore we have the following:

(i) $\bar{\gamma}_{\theta} \in C^{\infty}((0,1]) \cap C([0,1])$ with $\bar{\gamma}_{\theta}(0)=0$;

(ii) if $\theta_{1}<\theta_{2}$, then $\bar{\gamma}_{\theta_{1}}<\bar{\gamma}_{\theta_{2}}$ in $(0,1]$. 
To construct a candidate solution for all $\theta \geq 0$, we extend $\gamma_{\theta}$ by 0 to $(0,1)$, i.e., we set $\gamma_{\theta}(r)=0$ if $r \in\left(0, c_{\theta}\right]$, and we patch $\gamma_{\theta}$ and $\bar{\gamma}_{\theta}$ together by defining

$$
\gamma(r, \theta):= \begin{cases}0 & \text { if } \theta \in[0,1], \\ \gamma_{\theta}(r) & \text { if } \theta \in\left(1, \theta_{\lambda}\right), \\ \gamma_{\theta}(r) & \text { if } \theta \geq \theta_{\lambda} .\end{cases}
$$

The resulting function turns out to be the right candidate.

THEOREM 5.3. The function $\gamma$ defined by (5.7) is the unique solution of (4.3) in the sense of Definition 4.1. Moreover, $\gamma \in \operatorname{Lip}([0,+\infty) ; H)$.

In the rest of this section we prove Lemmas 5.1 and 5.2 and Theorem 5.3.

Proof of Lemma 5.1. We introduce $\gamma_{(0)}, \gamma_{(1)}$, and $\gamma_{(2)}$ as the solutions of the following auxiliary problems:

$$
\left\{\begin{array} { l } 
{ \mathcal { L } \gamma _ { ( 0 ) } ( r ) = 1 , } \\
{ \gamma _ { ( 0 ) } ( 1 ) = \gamma _ { ( 0 ) } ^ { \prime } ( 1 ) = 0 , }
\end{array} \quad \left\{\begin{array} { l } 
{ \mathcal { L } \gamma _ { ( 1 ) } ( r ) = - r , } \\
{ \gamma _ { ( 1 ) } ( 1 ) = \gamma _ { ( 1 ) } ^ { \prime } ( 1 ) = 0 , }
\end{array} \quad \left\{\begin{array}{l}
\mathcal{L} \gamma_{(2)}(r)=0, \\
\gamma_{(2)}(1)=1, \gamma_{(2)}^{\prime}(1)=-\frac{1}{2} .
\end{array}\right.\right.\right.
$$

It follows easily by comparison (see, e.g., the proof of (iv)) that $\gamma_{(0)},-\gamma_{(1)}$ and $\gamma_{(2)}$ are positive, decreasing, and convex in $(0,1)$. If a pair $(c, \gamma)$, with $c>0$, is a solution of $\left(\mathscr{P}_{\theta}\right)$, then

$$
\gamma=\gamma_{(0)}+\theta \gamma_{(1)}+\alpha \gamma_{(2)}
$$

for some $\alpha \in \mathbb{R}$, and the boundary conditions $(5.5)_{2}$ imply

$$
\left\{\begin{array}{l}
\theta \gamma_{(1)}(c)+\alpha \gamma_{(2)}(c)=-\gamma_{(0)}(c), \\
\theta \gamma_{(1)}^{\prime}(c)+\alpha \gamma_{(2)}^{\prime}(c)=-\gamma_{(0)}^{\prime}(c),
\end{array}\right.
$$

and vice versa, if $\alpha$ and $c>0$ are such that (5.10) holds, then $(c, \gamma)$, with $\gamma$ given by $(5.9)$, is a solution of $\left(\mathscr{P}_{\theta}\right)$. We now fix $c \in(0,1)$ and consider $(5.10)$ as a linear system in $(\theta, \alpha)$. The determinant of the system (5.10) is

$$
\delta(c):=\gamma_{(1)}(c) \gamma_{(2)}^{\prime}(c)-\gamma_{(2)}(c) \gamma_{(1)}^{\prime}(c) .
$$

Using (5.8), it is easily seen that $\delta$ satisfies

$$
\delta^{\prime}(c)=-\frac{\delta(c)}{c}+\frac{c \gamma_{(2)}(c)}{\lambda^{2}} \quad \text { in }(0,1), \quad \delta(1)=0,
$$

which may be integrated explicitly:

$$
\delta(c)=-\frac{1}{c \lambda^{2}} \int_{c}^{1} r^{2} \gamma_{(2)}(r) \mathrm{d} r \quad \text { for all } c \in(0,1] .
$$

Note that $\delta<0$ in $(0,1)$ since $\gamma_{(2)}>0$. Therefore, for any $c \in(0,1),(5.10)$ has a unique solution,

$$
\begin{aligned}
& \widehat{\theta}(c)=\frac{-\gamma_{(0)}(c) \gamma_{(2)}^{\prime}(c)+\gamma_{(2)}(c) \gamma_{(0)}^{\prime}(c)}{\delta(c)}=: \frac{\nu(c)}{\delta(c)}, \\
& \widehat{\alpha}(c)=\frac{-\gamma_{(1)}(c) \gamma_{(0)}^{\prime}(c)+\gamma_{(0)}(c) \gamma_{(1)}^{\prime}(c)}{\delta(c)}=: \frac{\xi(c)}{\delta(c)} .
\end{aligned}
$$


In order to invert $\widehat{\theta}$, with the help of (5.8) we notice that the numerator $\nu$ of $\widehat{\theta}$ solves

$$
\nu^{\prime}(c)=-\frac{\nu(c)}{c}+\frac{\gamma_{(2)}(c)}{\lambda^{2}} \text { in }(0,1), \quad \nu(1)=0,
$$

which, as before, may be integrated explicitly:

$$
\nu(c)=-\frac{1}{c \lambda^{2}} \int_{c}^{1} r \gamma_{(2)}(r) \mathrm{d} r \quad \text { for all } c \in(0,1] .
$$

Therefore

$$
\begin{gathered}
\widehat{\theta}^{\prime}(c)=\frac{\nu^{\prime}(c) \delta(c)-\nu(c) \delta^{\prime}(c)}{\delta^{2}(c)} \stackrel{(5.11),(5.14)}{=} \frac{\gamma_{(2)}(c)}{\lambda^{2} \delta^{2}(c)}(\delta(c)-c \nu(c)) \\
\stackrel{(5.12),(5.15)}{=}-c \gamma_{(2)}(c) \frac{\int_{c}^{1} r(r-c) \gamma_{(2)}(r) \mathrm{d} r}{\left(\int_{c}^{1} r^{2} \gamma_{(2)}(r) \mathrm{d} r\right)^{2}}<0 \text { for all } c \in(0,1),
\end{gathered}
$$

which implies that $\widehat{\theta}$ is invertible. We now notice that, letting $\hat{r}=r / \lambda$, the equation satisfied by $\gamma_{(2)}$ becomes the so-called modified Bessel equation of order 1 :

$$
\hat{r}^{2} \frac{d^{2}}{d \hat{r}^{2}} \gamma+\hat{r} \frac{d}{d \hat{r}} \gamma-\left(1+\hat{r}^{2}\right) \gamma=0,
$$

whose general solution is a linear combination of the modified Bessel functions $I_{1}(x)$ and $K_{1}(x)$; in particular, it is such that $\hat{r} \gamma_{(2)}(\hat{r}) \rightarrow C>0$ as $\hat{r} \rightarrow 0^{+}[1$, p. 374, section 9.6.1, and p. 375, sections 9.6.7-8]. Then

$$
\lim _{c \rightarrow 0^{+}} \widehat{\theta}^{\prime}(c)=-C_{2}<0
$$

and, after simple computations using de l'Hôpital's rule,

$$
\lim _{c \rightarrow 1^{-}} \widehat{\theta}^{\prime}(c)=-\gamma_{(2)}(1) \lim _{c \rightarrow 1^{-}} \frac{-\int_{c}^{1} r \gamma_{(2)}(r) \mathrm{d} r}{-2 c^{2} \gamma_{(2)}(c)\left(\int_{c}^{1} r^{2} \gamma_{(2)}(r) \mathrm{d} r\right)}=-\frac{1}{2} .
$$

Since (by (5.16)) $\widehat{\theta}^{\prime}$ is continuous in $(0,1),(5.17)$ and (5.18) imply that

$$
\widehat{\theta}^{\prime}(c) \leq-C_{1}<0 \text { for all } c \in(0,1) .
$$

In addition, recalling (5.13), (5.12), and (5.15),

$\lim _{c \rightarrow 1^{-}} \widehat{\theta}(c)=\lim _{c \rightarrow 1^{-}} \frac{\int_{c}^{1} s \gamma_{(2)}(s) \mathrm{ds}}{\int_{c}^{1} s^{2} \gamma_{(2)}(s) \mathrm{ds}}=1 \quad$ and $\quad \lim _{c \rightarrow 0^{+}} \widehat{\theta}(c)=\lim _{c \rightarrow 0^{+}} \frac{\int_{c}^{1} s \gamma_{(2)}(s) \mathrm{ds}}{\int_{c}^{1} s^{2} \gamma_{(2)}(s) \mathrm{ds}}=\theta_{\lambda} \in \mathbb{R}$.

Combining (5.19) and (5.20), we see that the function

$$
\widehat{\theta}^{-1}:\left(1, \theta_{\lambda}\right) \ni \theta \longmapsto c=c_{\theta} \in[0,1)
$$

is strictly decreasing and Lipschitz continuous: it uniquely determines the solution of $\left(\mathscr{P}_{\theta}\right)$,

$$
\gamma_{\theta}:=\gamma_{(0)}+\theta \gamma_{(1)}+\widehat{\alpha}\left(c_{\theta}\right) \gamma_{(2)}
$$


Since $c_{\theta}>0$, the regularity of $\gamma_{\theta}$ follows at once from that of $\gamma_{(0)}, \gamma_{(1)}$, and $\gamma_{(2)}$. In order to prove (ii)-(iv) we make three observations. First, differentiating $(5.10)_{1}$ with respect to $c$ and subtracting $(5.10)_{2}$, we obtain $\widehat{\theta}^{\prime}(c) \gamma_{(1)}(c)+\widehat{\alpha}^{\prime}(c) \gamma_{(2)}(c)=0$, whence

$$
\widehat{\alpha}^{\prime}(c)=-\widehat{\theta}^{\prime}(c) \frac{\gamma_{(1)}(c)}{\gamma_{(2)}(c)}, \quad \text { i.e., } \quad \frac{\mathrm{d}}{\mathrm{d} \theta} \widehat{\alpha}\left(c_{\theta}\right)=-\frac{\gamma_{(1)}(c)}{\gamma_{(2)}(c)}>0 .
$$

Combining (5.22) with (5.21) (evaluated at $r=1$ ) we obtain the following monotonicity property:

$$
1<\theta_{1}<\theta_{2}<\theta_{\lambda} \quad \Rightarrow \quad \gamma_{\theta_{1}}(1)<\gamma_{\theta_{2}}(1)
$$

Second,

$$
\gamma \text { cannot have a nonpositive local minimum in }(1 / \theta, 1) \text {. }
$$

Indeed, at a local minimum point $r_{0} \in(1 / \theta, 1)$, by (5.2) we would have $\gamma^{\prime \prime}\left(r_{0}\right)<0$, which is impossible. Third, $\gamma(1)>0$. Indeed, if $\gamma(1)<0$, then $\gamma^{\prime}(1)=-\gamma(1) / 2>0$, while if $\gamma(1)=0$, then $\gamma^{\prime}(1)=0$ and, as above, $\gamma^{\prime \prime}(1)<0$ (since $\theta>1$ ). Since $\gamma\left(c_{\theta}=0\right)$, both would contradict (5.24).

We are now ready to prove (ii)-(iv).

(ii) We first show that $c_{\theta}<1 / \theta$. We recall that $\gamma^{\prime}\left(c_{\theta}\right)=0$ and we note that $\gamma^{\prime \prime}\left(c_{\theta}\right)=1-\theta c_{\theta}$. If by contradiction $c_{\theta}>1 / \theta$, then $\gamma^{\prime \prime}\left(c_{\theta}\right)<0$; hence $\gamma$ would be negative in a right-neighborhood of $c_{\theta}$, in contradiction with $\gamma(1)>0$ and (5.24). If, instead, $c_{\theta}=1 / \theta$, then $\gamma^{\prime \prime}\left(c_{\theta}\right)=0$ : differentiating the equation, this implies that $\gamma^{\prime \prime \prime}\left(c_{\theta}\right)=-\theta<0$ and yields a contradiction as in the previous case.

(iii) We next show that $\gamma>0$ in $\left(c_{\theta}, 1\right]$. If not, since $\gamma\left(c_{\theta}\right)=0$ and $\gamma(1)>0$, by (5.24) $\gamma$ must have a nonpositive minimum point $r_{0} \in\left(c_{\theta}, 1 / \theta\right]$. On the other hand, by (ii) $\gamma^{\prime \prime}\left(c_{\theta}\right)=1-\theta c_{\theta}>0$; hence $\gamma$ is positive in a right-neighborhood of $c_{\theta}$. Since $\gamma\left(r_{0}\right) \leq 0, \gamma$ has a positive maximum in $r_{1} \in\left(c_{\theta}, r_{0}\right) \subseteq\left(c_{\theta}, 1 / \theta\right)$. But then $\gamma^{\prime \prime}\left(r_{1}\right)>1-\theta r_{1}>0$, a contradiction.

(iv) Finally, we show that (5.23) can be strengthened to

$$
1<\theta_{1}<\theta_{2}<\theta_{\lambda} \Rightarrow \gamma_{\theta_{1}}<\gamma_{\theta_{2}} \text { in }\left[c_{\theta_{1}}, 1\right] .
$$

Let $\bar{\theta}=\theta_{2}-\theta_{1}>0$. The difference $\bar{\gamma}=\gamma_{\theta_{2}}-\gamma_{\theta_{1}}$ satisfies

$$
\mathcal{L} \bar{\gamma}+\bar{\theta} r=0 \quad \text { in } \quad\left(c_{\theta_{1}}, 1\right], \quad \bar{\gamma}^{\prime}(1)+\frac{1}{2} \bar{\gamma}(1)=0 .
$$

Since $c_{\theta}$ is strictly decreasing, (iii) implies that $\bar{\gamma}\left(c_{\theta_{1}}\right)>0$. By (5.23) we also have $\bar{\gamma}(1)>0$. If $\bar{\gamma}$ had a nonpositive minimum at $r_{0} \in\left(c_{\theta_{1}}, 1\right)$, by $(5.25)_{1}$ we would have $\bar{\gamma}^{\prime \prime}\left(r_{0}\right)<0$, which is impossible. Hence $\bar{\gamma}>0$ in $\left[c_{\theta_{1}}, 1\right]$ and the proof is complete.

Proof of Lemma 5.2. Let $f \in H^{\prime}$ be defined by

$$
\langle f, \tilde{\gamma}\rangle:=\int_{0}^{1} \tilde{\gamma}(\theta r-1) r \mathrm{~d} r \quad \text { for all } \tilde{\gamma} \in H .
$$

According to Lemma 4.2, and to the Lax-Milgram theorem, there exists a unique function $\gamma=\bar{\gamma}_{\theta} \in H$ satisfying $a(\gamma, \tilde{\gamma})=\langle f, \tilde{\gamma}\rangle$ for all $\tilde{\gamma} \in H$, that is to say,

$$
\int_{0}^{1} \tilde{\gamma}(\theta r-1) r \mathrm{~d} r \stackrel{(4.10)}{=} \int_{0}^{1} \gamma \tilde{\gamma} r \mathrm{~d} r+\lambda^{2} \int_{0}^{1}\left(\gamma^{\prime} \tilde{\gamma}^{\prime}+\frac{\tilde{\gamma} \gamma}{r^{2}}\right) r \mathrm{~d} r+\frac{\gamma(1) \tilde{\gamma}(1)}{2}
$$


for all $\tilde{\gamma} \in H$. Choosing first $\tilde{\gamma} \in C_{c}^{\infty}((0,1))$ in (5.26), we see that $\gamma \in H_{\text {loc }}^{2}((0,1])$ and that

$$
\lambda^{2}\left(r \gamma^{\prime \prime}+\gamma^{\prime}-\frac{\gamma}{r}\right)-r \gamma=(1-\theta r) r \quad \text { a.e. in }(0,1),
$$

i.e., the equation in $\left(\mathscr{P}_{\theta}^{\prime}\right)$ holds. Choosing then $\tilde{\gamma} \in C_{c}^{\infty}((0,1])$ in (5.26), integrating by parts and using (5.27), we see that the boundary condition at $r=1$ in $\left(\mathscr{P}_{\theta}^{\prime}\right)$ holds, too. It follows immediately from linear ODE theory that $\bar{\gamma}_{\theta} \in C^{\infty}((0,1])$; together with (4.4), (i) holds. Finally, (ii) follows by comparison arguments as in the proof of Lemma 5.1, using that $\gamma(0, \theta)=0$.

Proof of Theorem 5.3. We extend the definition of $c_{\theta}$ with

$$
c_{\theta}= \begin{cases}1 & \text { if } \theta \in[0,1] \\ 0 & \text { if } \theta \geq \theta_{\lambda}\end{cases}
$$

We let $\gamma(\cdot)=\gamma(\cdot, \theta)$ and $c=c_{\theta}$ when no confusion arises. A few preliminary observations are in order. Let $a: H \times H \rightarrow \mathbb{R}$ be as in (4.6). We already know from Lemma 5.2 that

$$
a(\gamma, \tilde{\gamma})=\int_{0}^{1}(\theta r-1) \tilde{\gamma} r \mathrm{~d} r \quad \text { for all } \tilde{\gamma} \in H \quad \text { and all } \theta \geq \theta_{\lambda} .
$$

For $\theta \in\left(1, \theta_{\lambda}\right)$, we recall that $\gamma \in C^{\infty}([c, 1])$ is such that $\gamma=0$ in $(0, c)$ and

$$
\mathcal{L} \gamma=1-r \theta \quad \text { if } r \in(c, 1), \quad \gamma(c)=\gamma^{\prime}(c)=0, \quad \gamma^{\prime}(1)+\frac{1}{2} \gamma(1)=0 .
$$

Hence, for every $\tilde{\gamma} \in H$ we have

$$
\begin{aligned}
\int_{c}^{1}(r \theta-1) \tilde{\gamma} r \mathrm{~d} r & \stackrel{(5.29 a)}{=} \int_{c}^{1}(-\mathcal{L} \gamma) \tilde{\gamma} r \mathrm{~d} r \stackrel{(4.2)}{=} \int_{c}^{1}\left[-\lambda^{2}\left(\gamma^{\prime \prime} \tilde{\gamma} r+\gamma^{\prime} \tilde{\gamma}-\frac{\gamma \tilde{\gamma}}{r}\right)+\gamma \tilde{\gamma} r\right] \mathrm{d} r \\
& \stackrel{(5.29 b)}{=} \int_{c}^{1}\left[\lambda^{2}\left(\gamma^{\prime} \tilde{\gamma}^{\prime}+\frac{\gamma \tilde{\gamma}}{r^{2}}\right)+\gamma \tilde{\gamma}\right] r \mathrm{~d} r-\lambda^{2} \gamma^{\prime}(1) \tilde{\gamma}(1) \\
& \stackrel{(5.29 c)}{=} \int_{0}^{1}\left[\lambda^{2}\left(\gamma^{\prime} \tilde{\gamma}^{\prime}+\frac{\gamma \tilde{\gamma}}{r^{2}}\right)+\gamma \tilde{\gamma}\right] r \mathrm{~d} r+\lambda^{2} \frac{\gamma(1) \tilde{\gamma}(1)}{2} \stackrel{(4.10)}{=} a(\gamma, \tilde{\gamma}) .
\end{aligned}
$$

In view of (5.28), we conclude that

$$
a(\gamma, \tilde{\gamma})=\int_{c}^{1}(\theta r-1) \tilde{\gamma} r \mathrm{~d} r \quad \text { for all } \tilde{\gamma} \in H \text { and all } \theta \geq 0 .
$$

It follows from (ii) of Lemma 5.1 and (5.28) that

$$
c_{\theta}<1 / \theta \text { for all } \theta \in(0,+\infty) .
$$

We are now ready to complete the proof. First we show uniform Lipschitz continuity in $H$ :

$$
\left\|\gamma\left(\cdot, \theta_{2}\right)-\gamma\left(\cdot, \theta_{1}\right)\right\|_{H} \leq C\left|\theta_{2}-\theta_{1}\right| \quad \text { for all } 0 \leq \theta_{1} \leq \theta_{2} .
$$

Let $0 \leq \theta_{1}<\theta_{2}, \gamma_{i}(\cdot):=\gamma\left(\cdot, \theta_{i}\right)$, and $c_{i}:=c_{\theta_{i}}$. By (i) in Lemma 5.1, $c_{2}<c_{1}$. By Lemma 4.2,

$$
\begin{aligned}
\left\|\gamma_{2}-\gamma_{1}\right\|_{H}^{2} & \lesssim a\left(\gamma_{2}-\gamma_{1}, \gamma_{2}-\gamma_{1}\right) \\
& \stackrel{(5.30)}{=} \int_{0}^{1}\left(\theta_{2}-\theta_{1}\right)\left(\gamma_{2}-\gamma_{1}\right) r^{2} \mathrm{~d} r+\int_{c_{2}}^{c_{1}}\left(\theta_{1} r-1\right) \gamma_{2} r \mathrm{~d} r .
\end{aligned}
$$


In $\left(c_{2}, c_{1}\right)$, using (5.31) we have $\theta_{1} r-1 \leq \theta_{1} c_{1}-1<0$. Therefore

$$
\left\|\gamma_{2}-\gamma_{1}\right\|_{H}^{2} \lesssim\left(\theta_{2}-\theta_{1}\right)\left(\int_{0}^{1}\left(\frac{\gamma_{2}-\gamma_{1}}{r}\right)^{2} r \mathrm{~d} r\right)^{1 / 2} \leq\left\|\gamma_{2}-\gamma_{1}\right\|_{H}\left(\theta_{2}-\theta_{1}\right),
$$

which yields (5.32). Now (iv) of Lemma 5.1, (ii) of Lemma 5.2, and the definition of $\gamma$ imply that

$$
\dot{\gamma} \geq 0 \text { and } \dot{\gamma}(\cdot, \theta)=0 \text { in }\left(0, c_{\theta}\right) \text { if } c_{\theta}>0 \text {. }
$$

It remains for us to show (4.9). For a.e. $\theta \geq 0$, by (5.32) we have $\dot{\gamma} \in H$ : thus, for all $\tilde{\gamma} \in H$,

$$
\begin{aligned}
& a(\gamma, \tilde{\gamma}-\dot{\gamma}) \stackrel{(5.30)}{=} \quad \int_{0}^{1} \theta r^{2}(\tilde{\gamma}-\dot{\gamma}) \mathrm{d} r-\int_{0}^{c} \theta r^{2}(\tilde{\gamma}-\dot{\gamma}) \mathrm{d} r-\int_{c}^{1}(\tilde{\gamma}-\dot{\gamma}) r \mathrm{~d} r
\end{aligned}
$$

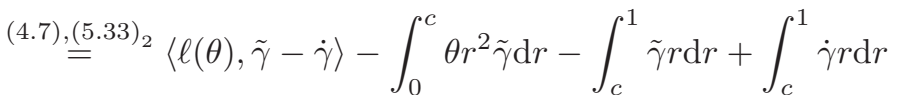

$$
\begin{aligned}
& \stackrel{(5.33)}{\geq}\langle\ell(\theta), \tilde{\gamma}-\dot{\gamma}\rangle-\int_{0}^{c} \theta r^{2}|\tilde{\gamma}| \mathrm{d} r-\int_{c}^{1}|\tilde{\gamma}| r \mathrm{~d} r+\int_{0}^{1}|\dot{\gamma}| r \mathrm{~d} r \\
& \stackrel{(5.31)}{\geq}\langle\ell(\theta), \tilde{\gamma}-\dot{\gamma}\rangle-\int_{0}^{1}|\tilde{\gamma}| r \mathrm{~d} r+\int_{0}^{1}|\dot{\gamma}| r \mathrm{~d} r .
\end{aligned}
$$

To prove uniqueness, let $\gamma_{1}$ and $\gamma_{2}$ be two solutions of (4.3) and let $\bar{\gamma}=\gamma_{1}-\gamma_{2}$. Then

$$
\begin{aligned}
\frac{\mathrm{d}}{\mathrm{d} \theta} \frac{a(\bar{\gamma}, \bar{\gamma})}{2} & =a(\bar{\gamma}, \dot{\bar{\gamma}}) \\
& =a\left(\gamma_{1}, \dot{\gamma}_{1}-\dot{\gamma}_{2}\right)-a\left(\gamma_{2}, \dot{\gamma}_{1}-\dot{\gamma}_{2}\right)=-a\left(\gamma_{1}, \dot{\gamma}_{2}-\dot{\gamma}_{1}\right)-a\left(\gamma_{2}, \dot{\gamma}_{1}-\dot{\gamma}_{2}\right) \\
& \leq-\left\langle\ell(\theta), \dot{\gamma}_{2}-\dot{\gamma}_{1}\right\rangle+\mathscr{D}\left(\dot{\gamma}_{2}\right)-\mathscr{D}\left(\dot{\gamma}_{1}\right)-\left\langle\ell(\theta), \dot{\gamma}_{1}-\dot{\gamma}_{2}\right\rangle+\mathscr{D}\left(\dot{\gamma}_{1}\right)-\mathscr{D}\left(\dot{\gamma}_{2}\right)=0,
\end{aligned}
$$

and the result follows from the coercivity of $a(\cdot, \cdot)$.

6. Formal asymptotic for $\boldsymbol{\lambda} \ll 1$. For a fixed $\theta$, we let $\gamma(\cdot):=\gamma(\cdot, \theta)$ denote the solution characterized in Theorem 5.3, and $c_{\theta}$ as in Lemma 5.1.

6.1. The bulk. We expand $\gamma$ and (for $\theta<\theta_{\lambda}$ ) $c_{\theta}$ in powers of $\lambda \ll 1$ : $\gamma=$ $\gamma_{0}+\lambda \gamma_{1}+\cdots$ and $c_{\theta}=c_{0}+\lambda c_{1}+\cdots$. At leading order, we see from (5.5) and (5.6) that $\gamma_{0}(r)=(\theta r-1)_{+}$and $c_{0}=1 / \theta$. Due to the incompatibility of $\gamma_{0}$ with the boundary conditions at $r=1 / \theta$ and at $r=1$, a boundary layer will form near each of the two points. In this section we address these local behaviors, and we use the former to determine the leading order value of the torque for $\lambda \ll 1$.

6.2. The boundary layer near the free boundary. For $\theta<\theta_{\lambda}$, we zoom into the free boundary with the help of the change of variables

$$
\left.\gamma(r)=\lambda g(x), \quad x:=\left(r-c_{\theta}\right) / \lambda \quad \text { (i.e., } r=c_{\theta}+\lambda x \text { and } \frac{d}{d x}=\lambda \frac{d}{d r}\right),
$$

which leaves the slope invariant: $\gamma^{\prime}(r)=g_{x}(x)$. Therefore we will use

$$
\theta=\gamma_{0}^{\prime}\left(r_{0}\right)=\lim _{x \rightarrow+\infty} g_{x}(x) \text { for all } r_{0} \in(1 / \theta, 1)
$$


in order to match $g$ with the bulk solution, $\gamma_{0}$. Neglecting the condition at $r=1$, (5.5) reads as

$$
\left\{\begin{array}{l}
g_{x x}+\lambda\left(c_{\theta}+\lambda x\right)^{-1} g_{x}-\lambda^{2}\left(c_{\theta}+\lambda x\right)^{-2} g-g=\frac{1}{\lambda}\left(1-\theta\left(c_{\theta}+\lambda x\right)\right), \\
g(0)=g_{x}(0)=0 .
\end{array}\right.
$$

We expand $g$ and $c$ in powers of $\lambda: g=g_{0}+\lambda g_{1}+\cdots$ and $c=1 / \theta+\lambda c_{1}+\cdots$. At leading order in $\lambda$ we have

$$
\left\{\begin{array}{l}
\left(g_{0}\right)_{x x}-g_{0}=\frac{1}{\lambda}\left(1-\theta\left(c_{0}+\lambda c_{1}+\lambda x\right)\right)=-\theta\left(c_{1}+x\right), \\
g_{0}(0)=\left(g_{0}\right)_{x}(0)=0
\end{array}\right.
$$

The general solution of $(6.3)_{1}$ is $g_{0}=\theta\left(c_{1}+x\right)+a e^{x}+b e^{-x}$; the initial conditions $(6.3)_{2}$ yield

$$
g_{0}=\theta\left(c_{1}+x\right)-\frac{1}{2} \theta\left(1+c_{1}\right) e^{x}+\frac{1}{2} \theta\left(1-c_{1}\right) e^{-x}
$$

Using the matching condition (6.1) yields $c_{1}=-1$. Therefore

(6.4) $c_{\theta}=\theta^{-1}-\lambda+O\left(\lambda^{2}\right)$ and $g=\theta\left(x-1+e^{-x}\right)+O(\lambda)$ for $\lambda \ll 1$ and $\theta<\theta_{\lambda}$,

the former coinciding with (1.19).

As we will see, in order to quantify the dependence of the torque on $\lambda$, we need to work out the next order correction to $c_{\theta}$. It follows from (6.2) and (6.4) that

$$
\left(g_{1}\right)_{x x}+\theta\left(g_{0}\right)_{x}-g_{1}=-\theta c_{2}, \quad g_{1}(0)=\left(g_{1}\right)_{x}(0)=0 .
$$

The general solution of the $\mathrm{ODE}(6.5)_{1}$ is $g_{1}=a e^{x}+b e^{-x}+\theta^{2}+\theta c_{2}-\theta^{2} x e^{-x} / 2$; the initial conditions $(6.5)_{2}$ yield

$$
g_{1}=-\left(\frac{1}{4} \theta^{2}+\frac{1}{2} \theta c_{2}\right) e^{x}-\left(\frac{3}{4} \theta^{2}+\frac{1}{2} \theta c_{2}\right) e^{-x}+\theta^{2}+\theta c_{2}-\frac{1}{2} \theta^{2} x e^{-x} .
$$

The matching condition $(6.1)$ requires $\left(g_{1}\right)_{x}$ be bounded as $x \rightarrow+\infty$. Hence $c_{2}=$ $-\theta / 2$ and

$$
c_{\theta} \sim \theta^{-1}-\lambda-\frac{1}{2} \theta \lambda^{2} \text { for } \lambda \ll 1 \text { and } \theta<\theta_{\lambda} \text {. }
$$

6.3. The boundary layer near $\boldsymbol{r}=1$. Here we motivate the expansion (1.20). For $\theta>1$, we zoom into $r=1$ with the help of the change of variables

$$
\gamma(r)=\theta-1+\lambda g(x), \quad x=(1-r) / \lambda \quad\left(\text { i.e., } r=1-\lambda x \text { and } \frac{d}{d x}=-\lambda \frac{d}{d r}\right),
$$

which again leaves the slope invariant (up to the sign). Therefore we will use as matching condition

$$
\lim _{x \rightarrow+\infty} g_{x}(x)=-\theta
$$

Neglecting the boundary conditions at $r=c_{\theta}$, it follows from (5.5) and (5.6) that

$$
\left\{\begin{array}{l}
g_{x x}-\lambda(1-\lambda x)^{-1} g_{x}-\lambda^{2}(1-\lambda x)^{-2} g-g=\theta x+\lambda(1-\lambda x)^{-2}(\theta-1) \\
g_{x}(0)=\frac{1}{2}(\theta-1+\lambda g(0))
\end{array}\right.
$$


We expand $g$ in powers of $\lambda: g=g_{0}+\lambda g_{1}+\cdots$. At leading order in $\lambda$ we have

$$
\left(g_{0}\right)_{x x}-g_{0}=\theta x, \quad g_{0}^{\prime}(0)=\frac{1}{2}(\theta-1) .
$$

The general solution and the matching condition yield $g_{0}=-\theta x+\frac{1}{2}(1-3 \theta) e^{-x}$. In terms of the original variables,

$$
\gamma \sim \theta-1-\lambda \theta x+\frac{1}{2} \lambda(1-3 \theta) e^{-x}=\theta-1-\theta(1-r)+\frac{1}{2} \lambda(1-3 \theta) e^{-\frac{1-r}{\lambda}}
$$

and (1.20) follows. In particular,

$$
\gamma(1) \sim \theta-1-\frac{1}{2} \lambda(3 \theta-1) \quad \text { for } \lambda \ll 1 .
$$

6.4. The asymptotics for the torque. For $1<\theta<\theta_{\lambda}$, we have

$$
\begin{aligned}
q(\theta) & \stackrel{(1.17)}{=} 3 \int_{0}^{1}(\theta r-\gamma) r^{2} \mathrm{~d} r \stackrel{(4.3)}{=} 3 \int_{0}^{c_{\theta}} \theta r^{3} \mathrm{~d} r+3 \int_{c_{\theta}}^{1}\left(r^{2}-\lambda^{2}\left(r^{2} \gamma^{\prime \prime}+r \gamma^{\prime}-\gamma\right)\right) \mathrm{d} r \\
& =\frac{3}{4} \theta c_{\theta}^{4}+1-c_{\theta}^{3}-3 \lambda^{2}\left(\left[r^{2} \gamma^{\prime}\right]_{c_{\theta}}^{1}-\int_{c_{\theta}}^{1}\left(r \gamma^{\prime}+\gamma\right) \mathrm{d} r\right) \\
& \stackrel{(4.3)}{=} 1-\frac{1}{4} c_{\theta}^{3}\left(4-3 c_{\theta} \theta\right)+\frac{9}{2} \lambda^{2} \gamma(1) .
\end{aligned}
$$

For $\theta>\theta_{\lambda}$, we have instead

$$
q(\theta) \stackrel{(1.17)}{=} 3 \int_{0}^{1}(\theta r-\gamma) r^{2} \mathrm{~d} r \stackrel{(4.3)}{=} 3 \int_{0}^{1}\left(r^{2}-\lambda^{2}\left(r^{2} \gamma^{\prime \prime}+r \gamma^{\prime}-\gamma\right)\right) \mathrm{d} r \stackrel{(4.3)}{=} 1+\frac{9}{2} \lambda^{2} \gamma(1) .
$$

Plugging (6.6) and (6.7) into the form $\gamma(1) \sim \theta-1$ yields, after straightforward computations,

$$
q(\theta) \sim\left\{\begin{array}{ll}
1-\frac{1}{4 \theta^{3}}+\frac{3 \lambda^{2}}{2 \theta}+\frac{9}{2} \lambda^{2}(\theta-1) & \text { if } \theta \in\left(1, \theta_{\lambda}\right), \\
1+\frac{9 \lambda^{2}}{2}(\theta-1) & \text { if } \theta>\theta_{\lambda},
\end{array} \quad \text { for } \lambda \ll 1 .\right.
$$

Note that the $O(\lambda)$-term in the expansion for $\theta<\theta_{\lambda}$ vanishes, which points to the aforementioned necessity of a second-order expansion of $c_{\theta}$. Since $\gamma \in \operatorname{Lip}([0, \infty) ; H)$, $q$ need be continuous across $\theta=\theta_{\lambda}$. Therefore, (1.21) and (1.22) follow from

$$
-\frac{1}{4 \theta_{\lambda}^{3}}+\frac{3 \lambda^{2}}{2 \theta_{\lambda}} \ll 1 \Longleftrightarrow \theta_{\lambda} \sim \frac{1}{\sqrt{6} \lambda} .
$$

7. Plastic spin. In the small-strain theory proposed by Gurtin in [20], the plastic distortion $\mathbf{H}^{\mathrm{p}}:=\nabla \mathbf{u}-\mathbf{H}^{\mathrm{e}}$ is not symmetric:

$$
\mathbf{H}^{\mathrm{p}}=\mathbf{E}^{\mathrm{p}}+\mathbf{W}^{\mathrm{p}}, \quad \mathbf{E}^{\mathrm{p}} \quad \text { symmetric }, \quad \mathbf{W}^{\mathrm{p}} \quad \text { skew-symmetric. }
$$

The defect energy and effective flow rate considered in [20] are

$$
\psi_{\mathrm{d}}=\frac{1}{2} \mu L^{2}\left|\operatorname{curl}^{\mathrm{p}}\right|^{2}, \quad \text { respectively, } \quad d^{\mathrm{p}}=\sqrt{|\dot{\mathbf{E}}|^{2}+\chi\left|\dot{\mathbf{W}}^{\mathrm{p}}\right|^{2}+\ell^{2}|\nabla \dot{\mathbf{E}} \mathrm{p}|^{2}},
$$

where $\chi>0$ is a constitutive parameter that measures the importance of dissipation associated with plastic rotations (see also [5] for a discussion in the case of simple 
shear). Note that $(7.2)_{1}$ generalizes (1.4) in the particular case $\eta=0$. Within our working assumptions (1.6), Gurtin's theory leads to the following flow rule:

$$
\mathbf{T}_{0}+\mu L^{2}\left(\Delta \mathbf{H}^{\mathrm{p}}-\nabla \operatorname{div} \mathbf{H}^{\mathrm{p}}+\frac{1}{3}\left(\operatorname{div} \operatorname{div} \mathbf{H}^{\mathrm{p}}\right) \mathbf{I}\right) \in \partial \delta_{\chi}\left(\dot{\mathbf{H}}^{\mathrm{p}}\right),
$$

where now the dissipation is $\delta_{\chi}\left(\dot{\mathbf{H}}^{\mathrm{p}}\right)=\sqrt{2} k \sqrt{|\dot{\mathbf{E}} \mathrm{p}|^{2}+\chi\left|\dot{\mathbf{W}}^{\mathrm{p}}\right|^{2}}$ and

$$
\partial \delta_{\chi}\left(\dot{\mathbf{H}}^{\mathrm{p}}\right):=\left\{\mathbf{A}_{0} \in \mathbb{R}_{0}^{3 \times 3}: \delta_{\chi}\left(\widetilde{\mathbf{H}}^{\mathrm{p}}\right)-\delta_{\chi}\left(\dot{\mathbf{H}}^{\mathrm{p}}\right) \geq \mathbf{A}_{0}:\left(\widetilde{\mathbf{H}}^{\mathrm{p}}-\dot{\mathbf{H}}^{\mathrm{p}}\right) \text { for all } \widetilde{\mathbf{H}}^{\mathrm{p}} \in \mathbb{R}_{0}^{3 \times 3}\right\} .
$$

As announced in the introduction, a solution of (7.3) is readily constructed by taking the solution of (1.8) for $\eta=0$ and by setting $\mathbf{W}^{\mathrm{p}}=0$. Indeed, if $\mathbf{E}^{\mathrm{p}}$ is given by the ansatz (1.10), with $\gamma^{\mathrm{p}}$ solving (3.5)-(3.6) with $\eta=0$ and $\mathbf{W}^{\mathrm{p}}=0$, then

$$
\begin{aligned}
& \partial \delta_{\chi}\left(\dot{\mathbf{H}}^{\mathrm{p}}\right)=\partial \delta_{\chi}\left(\dot{\mathbf{E}}^{\mathrm{p}}\right) \\
& :=\left\{\mathbf{A}_{0} \in \mathbb{R}_{0}^{3 \times 3}: \delta_{\chi}\left(\widetilde{\mathbf{H}}^{\mathrm{p}}\right)-\delta_{\chi}\left(\dot{\mathbf{E}}^{\mathrm{p}}\right) \geq \mathbf{A}_{0}:\left(\widetilde{\mathbf{H}}^{\mathrm{p}}-\dot{\mathbf{E}}^{\mathrm{p}}\right) \text { for all } \widetilde{\mathbf{H}}^{\mathrm{p}} \in \mathbb{R}_{0}^{3 \times 3}\right\} .
\end{aligned}
$$

We decompose $\widetilde{\mathbf{H}}^{\mathrm{p}}$ as in (7.1) and we use that $\delta_{\chi}\left(\dot{\mathbf{E}}^{\mathrm{p}}\right)=\delta\left(\dot{\mathbf{E}}^{\mathrm{p}}\right)$, that $\delta\left(\widetilde{\mathbf{E}}^{\mathrm{p}}\right)=\delta\left(\widetilde{\mathbf{H}}^{\mathrm{p}}\right) \leq$ $\delta_{\chi}\left(\widetilde{\mathbf{H}}^{\mathrm{p}}\right)$, and that $\mathbf{A}_{0}: \widetilde{\mathbf{H}}^{\mathrm{p}}=\mathbf{A}_{0}: \widetilde{\mathbf{E}}^{\mathrm{p}}$ if $\mathbf{A}_{0} \in \mathbb{R}_{0, \text { sym }}^{3 \times 3}$. Then

$$
\begin{aligned}
& \partial \delta_{\chi}\left(\dot{\mathbf{H}}^{\mathrm{p}}\right) \supset\left\{\mathbf{A}_{0} \in \mathbb{R}_{0, s y m}^{3 \times 3}: \delta\left(\widetilde{\mathbf{E}}^{\mathrm{p}}\right)-\delta\left(\dot{\mathbf{E}}^{\mathrm{p}}\right) \geq \mathbf{A}_{0}:\left(\widetilde{\mathbf{E}}^{\mathrm{p}}-\dot{\mathbf{E}}^{\mathrm{p}}\right) \text { for all } \widetilde{\mathbf{H}}^{\mathrm{p}} \in \mathbb{R}_{0}^{3 \times 3}\right\} \\
&=\left\{\mathbf{A}_{0} \in \mathbb{R}_{0, s y m}^{3 \times 3}: \delta\left(\widetilde{\mathbf{E}}^{\mathrm{p}}\right)-\delta\left(\dot{\mathbf{E}}^{\mathrm{p}}\right) \geq \mathbf{A}_{0}:\left(\widetilde{\mathbf{E}}^{\mathrm{p}}-\dot{\mathbf{E}}^{\mathrm{p}}\right) \text { for all } \widetilde{\mathbf{E}}^{\mathrm{p}} \in \mathbb{R}_{0, s y m}^{3 \times 3}\right\} \\
& \stackrel{(1.7)}{=} \partial \delta\left(\dot{\mathbf{E}}^{\mathrm{p}}\right) \stackrel{(3.8)}{\ni} \mathbf{T}_{0}+\mu L^{2} \Delta \mathbf{E}^{\mathrm{p}} \stackrel{(3.7)}{\ni} \mathbf{T}_{0}+\mu L^{2}\left(\Delta \mathbf{E}^{\mathrm{p}}-\nabla \operatorname{div} \mathbf{E}^{\mathrm{p}}+\frac{1}{3}\left(\operatorname{div} \operatorname{div} \mathbf{E}^{\mathrm{p}}\right) \mathbf{I}\right) \\
&=\mathbf{T}_{0}+\mu L^{2}\left(\Delta \mathbf{H}^{\mathrm{p}}-\nabla \operatorname{div} \mathbf{H}^{\mathrm{p}}+\frac{1}{3}\left(\operatorname{div} \operatorname{div} \mathbf{H}^{\mathrm{p}}\right) \mathbf{I}\right) \quad\left(\text { since } \mathbf{W}^{\mathrm{p}}=0\right) .
\end{aligned}
$$

Acknowledgments. The authors thank the anonymous reviewers for their comments and suggestions, and for pointing out additional references on gradient plasticity. GT also thanks Lorenzo Bardella for several useful discussions.

\section{REFERENCES}

[1] M. Abramowitz and I. A. Stegun, eds., Handbook of Mathematical Functions with Formulas, Graphs, and Mathematical Tables, U.S. Government Printing Office, Washington, D.C., 1964.

[2] A. Acharya And J. L. Bassani, Lattice incompatibility and a gradient theory of crystal plasticity, J. Mech. Phys. Solids, 48 (2000), pp. 1565-1595.

[3] E. C. Aifantis, On the microstructural origin of certain inelastic models, J. Eng. Mat. Tech., 106 (1984), pp. 326-330.

[4] L. Anand, M. E. Gurtin, S. P. Lele, and C. Gething, A one-dimensional theory of straingradient plasticity: Formulation, analysis, numerical results, J. Mech. Phys. Solids, 53 (2005), pp. 1789-1826.

[5] L. BARdella, A comparison between crystal and isotropic strain gradient plasticity theories with accent on the role of the plastic spin, Euro. J. Mech. A-Solids, 28 (2009), pp. 638-646.

[6] M. Bertsch, R. Dal Passo, L. Giacomelli, and G. Tomassetti, A nonlocal and fully nonlinear degenerate parabolic system from strain-gradient plasticity, Discrete Contin. Dyn. Syst. Ser. B, 15 (2011), pp. 15-43.

[7] G. Borino And C. Polizzotto, Thermodynamically consistent residual-based gradient plasticity theory and comparisons, Modelling Simul. Mater. Sci. Eng., 15 (2007), pp. S23-S35.

[8] O. W. Dillon and J. Kratochvíl, A strain gradient theory of plasticity, Int. J. Solids Struct., 6 (1970), pp. 1513-1533.

[9] D. Dunstan, B. Ehrler, R. Bossis, S. Joly, K. P'NG, And A. Bushby, Elastic limit and strain hardening of thin wires in torsion, Phys. Rev. Lett., 103 (2009), pp. 1-4. 
[10] F. Ebobisse And P. NeFF, Existence and uniqueness for rate-independent infinitesimal gradient plasticity with isotropic hardening and plastic spin, Math. Mech. Solids, 15 (2010), pp. 691703.

[11] N. A. Fleck And J. W. Hutchinson, Strain gradient plasticity, Adv. Appl. Mech., 33 (1997), pp. 295-361.

[12] N. A. Fleck and J. W. Hutchinson, A reformulation of strain gradient plasticity, J. Mech. Phys. Solids, 49 (2001), pp. 2245-2271.

[13] N. A. Fleck, G. M. Muller, M. F. Ashby, and J. W. Hutchinson, Strain gradient plasticity: Theory and experiment, Acta Metall. Mater., 42 (1994), pp. 475-487.

[14] N. A. FleCK AND J. R. Willis, A mathematical basis for strain-gradient plasticity theory. I. Scalar plastic multiplier, J. Mech. Phys. Solids, 57 (2009), pp. 161-177.

[15] A. Garroni, G. Leoni, and M. Ponsiglione, Gradient theory for plasticity via homogenization of discrete dislocations, J. Eur. Math. Soc. (JEMS), 12 (2010), pp. 1231-1266.

[16] A. Giacomini AND L. Lussardi, Quasi-static evolution for a model in strain gradient plasticity, SIAM J. Math. Anal., 40 (2008), pp. 1201-1245.

[17] P. Gudmundson, A unified treatment of strain gradient plasticity, J. Mech. Phys. Solids, 52 (2004), pp. 1379-1406.

[18] M. E. GURTIN AND L. ANAND, Thermodynamics applied to gradient theories involving the accumulated plastic strain: The theories of Aifantis and Fleck and Hutchinson and their generalization, J. Mech. Phys. Solids, 57 (2009), pp. 405-421.

[19] M. E. Gurtin, The Linear Theory of Elasticity, in Handbuch der Physik, S. Flügge, ed., Vol. VIa/2, Springer Verlag, Berlin, 1972.

[20] M. E. GurTin, A gradient theory of small-deformation isotropic plasticity that accounts for the Burgers vector and for dissipation due to plastic spin, J. Mech. Phys. Solids, 52 (2004), pp. 2545-2568.

[21] M. E. Gurtin And L. Anand, A theory of strain-gradient plasticity for isotropic, plastically irrotational materials. I. Small deformations, J. Mech. Phys. Solids, 53 (2005), pp. 16241649.

[22] M. E. Gurtin, E. Fried, And L. Anand, The Mechanics and Thermodynamics of Continua, Cambridge University Press, Cambridge, UK, 2010.

[23] W. Han and B. Daya Reddy, Plasticity. Mathematical Theory and Numerical Analysis, Springer-Verlag, New York, 1999.

[24] M. I. Idiart And N. A. Fleck, Size effects in the torsion of thin metal wires, Modelling Simul. Mater. Sci. Eng., 18 (2010), p. 015009.

[25] A. Mielke, Evolution of Rate-Independent Systems, in Evolutionary Equations. Vol. II, Handb. Differ. Eq., Elsevier/North-Holland, Amsterdam, 2005, pp. 461-559.

[26] P. NefF, Uniqueness of Strong Solutions in Infinitesimal Perfect Gradient Plasticity with Plastic Spin, in IUTAM-Symposium on Theoretical, Modelling and Computational Aspects of Inelastic Media (Cape Town, 2008), B. D. Reddy, ed., Springer, Berlin, 2008, pp. 129140.

[27] P. Neff, K. Chemmiński, And H. D. Alber, Notes on strain gradient plasticity. Finite strain covariant modelling and global existence in the infinitesimal rate-independent case, Math. Mod. Meth. Appl. Sci. (M3AS), 19 (2009), pp. 1-40.

[28] P. NefF, D. Pauly, And K.-J. Witsch, A canonical extension of Korn's first inequality to $H$ (Curl) motivated by gradient plasticity with plastic spin, C. R. Math. Acad. Sci. Paris, 349 (2011), pp. 1251-1254.

[29] P. NefF, A. Sydow, And C. Wieners, Numerical approximation of incremental infinitesimal gradient plasticity, Internat. J. Numer. Methods Engrg., 77 (2009), pp. 414-436.

[30] B. D. Reddy, F. Ebobisse, And A. McBride, Well-posedness of a model of strain gradient plasticity for plastically irrotational materials, Int. J. Plasticity, 24 (2008), pp. 55-73. 